\title{
Wireless Multicarrier Communications via Multipulse Gabor Riesz Bases
}

\author{
Manfred M. Hartmann, ${ }^{1,2}$ Gerald Matz, ${ }^{2}$ and Dieter Schafhuber ${ }^{3}$ \\ ${ }^{1}$ ARC Seibersdorf Research GmbH, Donau-City-Straße 1, A-1220 Wien, Austria \\ ${ }^{2}$ Institute of Communications and Radio Frequency Engineering, Vienna University of Technology, Gußhausstraße 25/389, \\ A-1040 Wien, Austria \\ ${ }^{3}$ BMW Forschung und Technik GmbH, Hanauer Straße 46, D-80992 München, Germany
}

Received 29 September 2004; Revised 19 January 2005; Accepted 5 April 2005

\begin{abstract}
We introduce multipulse multicarrier (MPMC) modulation, a wireless communication scheme that augments traditional singlepulse multicarrier systems by using multiple pulses at the transmitter and the receiver. The mathematical foundation of MPMC systems is established by the novel concept of multipulse Gabor Riesz bases. We adapt Zak-Fourier domain tools previously developed for multiwindow Gabor frames to analyze and design (bi)orthogonal multipulse Gabor Riesz bases and the corresponding MPMC systems in a computationally efficient manner. Furthermore, explicit expressions for the interference power and the spectral efficiency in MPMC transmissions over time-varying multipath channels are derived. The superiority of MPMC modulation over single-pulse multicarrier systems is finally demonstrated via numerical simulations.
\end{abstract}

Copyright (c) 2006 Hindawi Publishing Corporation. All rights reserved.

\section{INTRODUCTION}

\subsection{Background and contributions}

Multicarrier modulation [1-3] is an attractive technique for high data-rate wireless communications. Cyclic prefix orthogonal frequency-division multiplexing (CP-OFDM) [4-7] is a multicarrier scheme that is being used or proposed for numerous wireless standards like WLAN (IEEE $802.11 \mathrm{a} / \mathrm{g} / \mathrm{n}$, HIPERLAN/2), broadband wireless access (IEEE 802.16), wireless personal area networks (IEEE 802.15), and digital audio and video broadcasting (DAB, DRM, DVB-T). Moreover, it is a promising candidate for mobile radio systems beyond 3G. Recently, pulse-shaping OFDM $[8,9]$ and biorthogonal frequency-division multiplexing [9-12] have been shown to be less susceptible to channel distortions than CP-OFDM. We unifyingly refer to all of the above schemes as single-pulse multicarrier (SPMC) systems.

In this paper, we develop the foundations of multipulse multicarrier (MPMC) modulation, a novel wireless communication scheme that extends SPMC systems by using multiple transmit and receive pulses [13]. MPMC modulation is similar in spirit to multiwindow Gabor expansions [14] that extend the Gabor expansion $[15,16]$ by using multiple windows. It establishes a unifying framework for the various SPMC systems discussed in Section 1.2 and features increased design freedom which can be used to optimize system performance (cf. $[17,18])$.
The specific contributions of the paper are as follows.

(i) In Section 2, we introduce and discuss MPMC modulation and point out its relation to existing multicarrier schemes. An equivalent MPMC system formulation in terms of the piecewise Zak transform and a 2D Fourier transform is presented in Section 3.

(ii) General linear modulation schemes are studied using the theory of Riesz bases in Section 4. Specializing this to the MPMC context leads us to the novel concept of multipulse Gabor Riesz bases (Section 5).

(iii) We derive explicit expressions for the interference power and the spectral efficiency of MPMC transmissions over time-varying multipath channels in Section 6.

(iv) Numerical examples regarding the design and performance of MPMC systems are provided in Section 7, including a comparison with SPMC systems.

Some conclusions are finally provided in Section 8. As a basis for the introduction of MPMC modulation, we next discuss SPMC systems in slightly more detail.

\subsection{Single-pulse multicarrier systems}

The modulator of an SPMC system with $K$ subcarriers maps the transmit symbols $a_{l, k}(l$ and $k$ denote symbol time and 


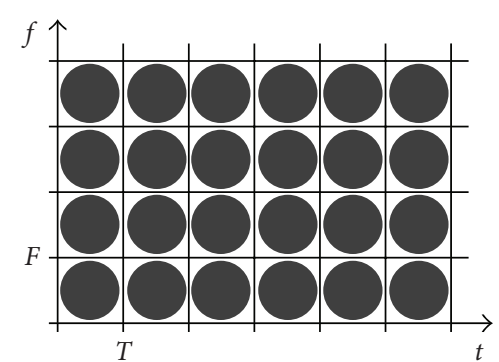

(a)

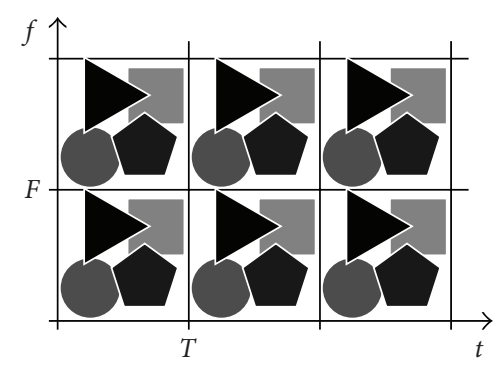

(b)

FIGURE 1: Schematic TF domain illustration of (a) an SPMC system and (b) an MPMC system with $M=4$.

subcarrier index, resp.) to the transmit signal

$$
s(t)=\sum_{l=-\infty}^{\infty} \sum_{k=0}^{K-1} a_{l, k}, g_{l, k}(t), \quad g_{l, k}(t) \triangleq g(t-l T) e^{j 2 \pi k F t} .
$$

Here, $g(t)$ is the prototype transmit pulse, and $T$ and $F$ denote the symbol duration and subcarrier spacing, respectively. It is seen that each symbol $a_{l, k}$ is carried by a pulse $g_{l, k}(t)$ localized about the time-frequency (TF) lattice point $(l T, k F)$ (cf. Figure 1(a)).

The SPMC demodulator calculates the receive sequence ${ }^{1}$

$$
x_{l, k}=\int_{t} r(t) \gamma_{l, k}^{*}(t) d t, \quad \gamma_{l, k}(t) \triangleq \gamma(t-l T) e^{j 2 \pi k F t},
$$

where $r(t)$ and $\gamma(t)$ denote the received signal and the prototype receive pulse, respectively.

For the case of an ideal channel $(r(t)=s(t))$, perfect symbol recovery $\left(x_{l, k}=a_{l, k}\right)$ is obtained if and only if the pulses $g(t), \gamma(t)$ and the lattice constants $T, F$ are designed such that the biorthogonality condition $\int_{t} g(t) \gamma_{l, k}^{*}(t) d t=\delta_{l} \delta_{k}$ is satisfied (cf. Section 4). For this, it is necessary that $T F \geq 1$ ( $T F$ is termed the redundancy). A larger redundancy amounts to a guard region between neighboring pulses which is beneficial for reducing intersymbol and intercarrier interference (ISCI)

\footnotetext{
${ }^{1}$ Integrals are from $-\infty$ to $\infty$ unless stated otherwise. Throughout the paper, the superscripts $*, T$, and $H$ denote the complex conjugate, transpose, and Hermitian transpose, respectively.
}

but reduces the number of symbols transmitted per second and Hertz.

Since wireless communication systems often operate over rapidly time-varying channels, minimizing ISCI is important to assure high spectral efficiencies. For SPMC systems, this problem leads to the development of pulse-shaping OFDM $[8,9]$ and BFDM [9-12]. Lattice OFDM [19] is another attempt to reduce ISCI by using a hexagonal TF lattice. Finally, OFDM/offset QAM (OFDM/OQAM) [9, 20-22] is an SPMC variant that allows to use critical redundancy $T F=1$ at the cost of increased equalization complexity.

\section{MULTIPULSE MULTICARRIER SYSTEMS}

\subsection{MPMC modulator and demodulator}

The fundamental idea behind MPMC systems is to use multiple transmit and receive prototype pulses [13] (see Figure 1(b)).

The MPMC modulator uses $M$ linearly independent prototype transmit pulses $g^{(m)}(t), m=1, \ldots, M$. We will refer to the vector $\mathbf{g}(t)=\left[g^{(1)}(t) \cdots g^{(M)}(t)\right]^{T}$ as transmit multipulse. At symbol time $l$ and subcarrier $k, M$ symbols $a_{l, k}^{(m)}, m=1, \ldots, M$, are transmitted in parallel via the pulses $g_{l, k}^{(m)}(t)=g^{(m)}(t-l T) e^{j 2 \pi k F t}, m=1, \ldots, M($ the symbol duration $T$ and the subcarrier spacing $F$ constitute the MPMC TF lattice parameters). With $K$ again denoting the number of subcarriers, the MPMC transmit signal equals

$$
s(t)=\sum_{m=1}^{M} \sum_{l=-\infty}^{\infty} \sum_{k=0}^{K-1} a_{l, k}^{(m)} g_{l, k}^{(m)}(t)
$$

Hence, MPMC modulation can be interpreted as superposition of $M$ SPMC modulators with different transmit pulses. Using the vector notations $\mathbf{a}_{l, k}=\left[a_{l, k}^{(1)} \cdots a_{l, k}^{(M)}\right]^{T}$, and $\mathbf{g}_{l, k}(t)=\left[g_{l, k}^{(1)}(t) \cdots g_{l, k}^{(M)}(t)\right]^{T}$, the MPMC transmit signal can be written as (cf. (1))

$$
s(t)=\sum_{l=-\infty}^{\infty} \sum_{k=0}^{K-1} \mathbf{a}_{l, k}^{T} \mathbf{g}_{l, k}(t)
$$

At the receiver, the MPMC demodulator employs a receive multipulse $\gamma(t)=\left[\gamma^{(1)}(t) \cdots \gamma^{(M)}(t)\right]^{T}$ consisting of $M$ linearly independent prototype receive pulses $\gamma^{(m)}(t)$, $m=1, \ldots, M$, to calculate the vector sequence $\mathbf{x}_{l, k}=$ $\left[x_{l, k}^{(1)} \cdots x_{l, k}^{(M)}\right]^{T}$ from the received signal $r(t)$ according to (cf. (2))

$$
\mathbf{x}_{l, k}=\int_{t} r(t) \boldsymbol{\gamma}_{l, k}^{*}(t) d t
$$

Here, $\gamma_{l, k}(t)=\left[\gamma_{l, k}^{(1)}(t) \cdots \gamma_{l, k}^{(M)}(t)\right]^{T}$ with $\gamma_{l, k}^{(m)}(t)=\gamma^{(m)}(t-$ $l T) e^{j 2 \pi k F t}$.

Block diagrams of MPMC modulator and demodulator are shown in Figure 2.

\subsection{Biorthogonality and TF lattice}

For MPMC systems, perfect symbol recovery $\left(\mathbf{x}_{l, k}=\mathbf{a}_{l, k}\right)$ in the case of an ideal channel $(r(t)=s(t))$ is obtained if and only 

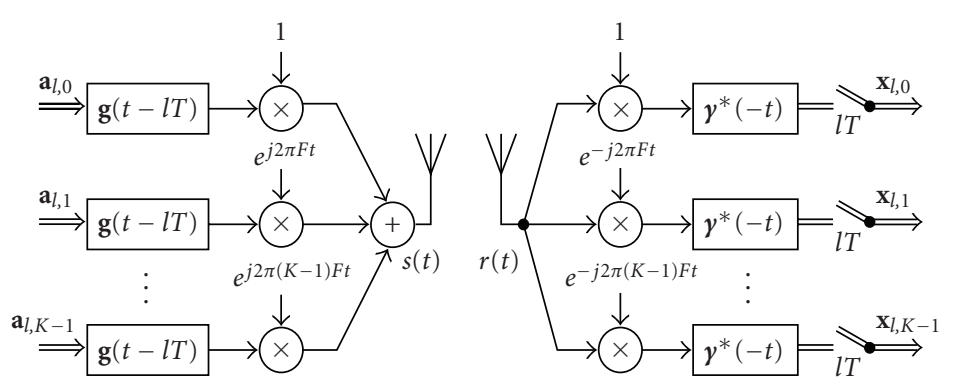

FIgURE 2: Block diagrams of MPMC modulator and demodulator.

if the multipulses $\mathbf{g}(t)$ and $\boldsymbol{\gamma}(t)$ and the TF lattice parameters $T$ and $F$ are chosen such that the biorthogonality condition

$$
\int_{t} \mathbf{g}(t) \boldsymbol{\gamma}_{l, k}^{H}(t) d t=\delta_{l} \delta_{k} \mathbf{I}
$$

is satisfied (i.e., $\left\{\mathbf{g}_{l, k}(t)\right\}$ and $\left\{\boldsymbol{\gamma}_{l, k}(t)\right\}$ are biorthogonal sets). If in addition $\mathbf{g}(t)=\boldsymbol{\gamma}(t)$, then $\left\{\mathbf{g}_{l, k}(t)\right\}$ is an orthogonal set. (Bi)orthogonal pulses require that the sets $\left\{\mathbf{g}_{l, k}(t)\right\},\left\{\gamma_{l, k}(t)\right\}$ constitute multipulse Gabor Riesz bases (see Section 5) which in turn presuppose that the redundancy $T F / M$ satisfies $T F / M \geq 1$, consistent with the single-pulse case. Thus, increasing the number of pulses $M$ requires a corresponding reduction of the TF lattice density (cf. Figure 1).

For fixed $M$, choosing the TF lattice parameters $T$ and $F$ is equivalent to specifiying the lattice ratio $T / F$ and the redundancy $T F / M$. Choosing the redundancy $T F / M$ is a tradeoff between high spectral efficiency (achieved with small redundancy) and robustness against ISI/ICI (obtained for large redundancy that reduces the overlap of adjacent pulses). Typically, $T F / M=1+\epsilon$ with $\epsilon=0.02 \cdots 0.5$ (according to the Balian-Low-type theorem discussed in Section 5, TF/M = 1 leads to poorly localized pulses). Regarding the lattice ratio, symmetry arguments suggest the choice $T / F=\tau_{\max } / \nu_{\max }$ (cf. $[10,19]$ for the case $M=1$ ), where $\tau_{\max }$ and $\nu_{\max }$ are the maximum delay and the maximum Doppler frequency, respectively, of the channel. The impact of the MPMC TF lattice on spectral efficiency will be illustrated in Section 6.

\subsection{Special cases}

Various existing SPMC systems fit within the MPMC framework as special cases. In particular, (pulse-shaping) OFDM $[3-5,8,9]$ and BFDM [9-12] are simple special cases with $M=1$.

Lattice OFDM [19], an SPMC scheme using an orthogonalized Gaussian pulse $g(t)$ on a hexagonal TF lattice (see Figure 3(a)), can be viewed as MPMC system with $M=2$ and transmit/receive multipulses $\mathbf{g}(t)=\boldsymbol{\gamma}(t)=[g(t) g(t-$ $\left.T / 2) e^{j \pi F t}\right]^{T}$ (cf. Figure 3(b)).

OFDM/OQAM $[9,20-22]$ can be interpreted as $M=4$ MPMC system with real-valued transmit symbols and transmit multipulse $\mathbf{g}(t)=\left[g(t) j g(t) e^{j \pi t / T} j g(t-T / 2) g(t-\right.$ $\left.T / 2) e^{j \pi t / T}\right]^{T}$ (see Figure 4(a)). While OFDM/OQAM uses

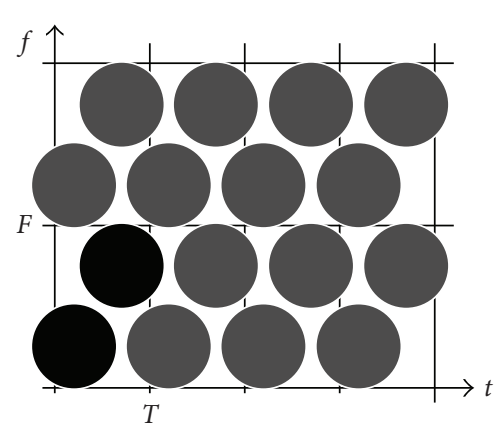

(a)

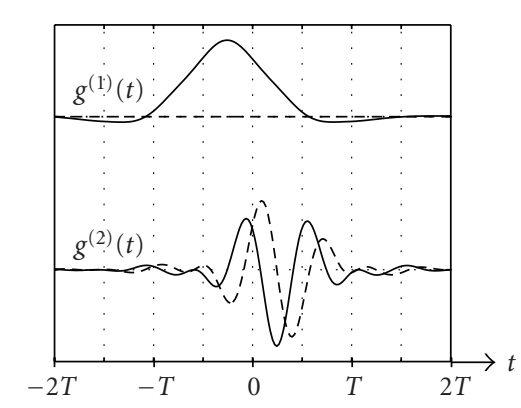

(b)

FIGURE 3: Lattice OFDM system: (a) equivalent MPMC representation with $M=2$, (b) corresponding MPMC multipulse for $T F / M=$ 1.5 (solid line: real part, dashed line: imaginary part).

$T F / M=1 / 2$, perfect symbol recovery for an ideal channel can still be achieved due to the restriction to real-valued symbols.

Finally, multicarrier direct sequence CDMA (MC-DSCDMA), a combination of OFDM and CDMA [23], can be interpreted as an MPMC system where the prototype pulses $\left\{g^{(m)}(t)\right\}, m=1, \ldots, M$, correspond to the M CDMA chip sequences (cf. Figure 4(b)).

\section{ZAK-FOURIER FORMULATION}

We next present an alternative MPMC system description in terms of the piecewise Zak transform [14] and a 2D Fourier 
transform. We note that the (piecewise) Zak transform has successfully been applied for the analysis and design of (multiwindow) Gabor frames $[15,16]$. This description applies to systems with rational lattice, $T F=p / q, p, q \in \mathbb{N}$ (note that this is not a severe restriction). For simplicity, we first consider the case of integer TF lattice, $T F=p \in \mathbb{N}$ and discuss the extension to rational TF lattice in Section 3.3.

\section{1. (Piecewise) Zak transform and 2D Fourier transform}

The Zak transform of a signal ${ }^{2} s(t) \in L^{2}(\mathbb{R})$ is defined as (cf. [14])

$$
(\mathcal{Z} s)(\eta, \theta) \triangleq \frac{1}{\sqrt{F}} \sum_{n=-\infty}^{\infty} s\left(\frac{\eta+n}{F}\right) e^{-j 2 \pi \theta n}
$$

In our context, $F$ is the MPMC subcarrier spacing. The Zak transform is (quasi-)periodic in the sense that

$$
(\mathcal{Z} s)(\eta+m, \theta+n)=e^{j 2 \pi \theta m}(\mathcal{Z} s)(\eta, \theta), \quad m, n \in \mathbb{Z} .
$$

Consequently, it is uniquely defined by its values on the unit square $(\eta, \theta) \in \mathcal{U}^{2}, \mathcal{U}=[0,1)$. The Zak transform can be inverted via the relation

$$
s\left(t+\frac{n}{F}\right)=\frac{1}{\sqrt{F}} \int_{0}^{1}(\mathcal{Z} s)(t F, \theta) e^{j 2 \pi \theta n} d \theta, \quad 0 \leq t<\frac{1}{F}, n \in \mathbb{Z},
$$

and it is a unitary mapping from $L^{2}(\mathbb{R})$ to the Hilbert space $L^{2}\left(U^{2}\right)$ with inner product

$$
\langle\mathcal{Z} s, \mathcal{Z} r\rangle_{L^{2}\left(u^{2}\right)} \triangleq \int_{0}^{1} \int_{0}^{1}(\mathcal{Z} r)^{*}(\eta, \theta)(\mathcal{Z} s)(\eta, \theta) d \eta d \theta .
$$

The Zak transform is covariant to time and frequency shifts in the sense that

$$
\begin{aligned}
& \widetilde{s}(t)=s(t-\tau) e^{j 2 \pi \nu t} \\
& \Longleftrightarrow(\mathcal{Z} \widetilde{s})(\eta, \theta)=e^{j 2 \pi \eta v / F}(\mathcal{Z} s)\left(\eta-\tau F, \theta-\frac{\nu}{F}\right) .
\end{aligned}
$$

For integer TF lattice, (8) and (11) imply that

$$
\begin{aligned}
& s_{l, k}(t)=s(t-l T) e^{j 2 \pi k F t} \\
& \Longleftrightarrow\left(\mathcal{Z}_{l, k}\right)(\eta, \theta)=e^{-j 2 \pi(l p \theta-k \eta)}(\mathcal{Z} s)(\eta, \theta) .
\end{aligned}
$$

The piecewise Zak transform (PZT) of $s(t)$ is defined as the length- $p$ vector [14]

$$
\begin{aligned}
& \left(\mathscr{Z}_{p} s\right)(\eta, \theta) \\
& \quad \triangleq\left[(\mathcal{Z} s)(\eta, \theta)(\mathcal{Z} s)\left(\eta, \theta+\frac{1}{p}\right) \cdots(\mathcal{Z} s)\left(\eta, \theta+\frac{p-1}{p}\right)\right]^{T} .
\end{aligned}
$$

\footnotetext{
2 The space of functions (sequences) that are square-integrable (squaresummable) on a set $\mathbb{S}$ are denoted by $L^{2}(\mathbb{S})\left(l^{2}(\mathbb{S})\right.$.
}

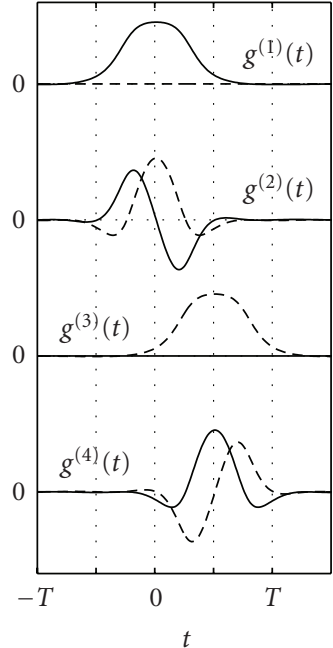

(a)

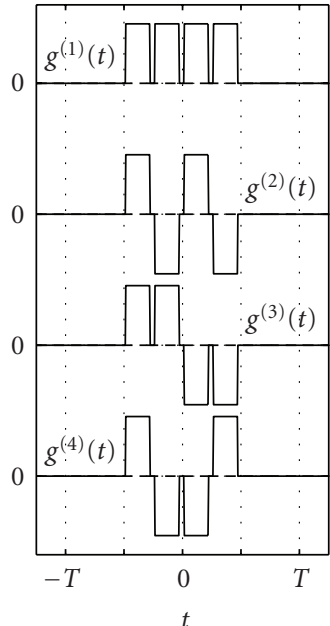

(b)
Figure 4: Multipulses for special cases of MPMC modulation: (a) OFDM/OQAM, (b) MC-DS-CDMA with $M=4$. (solid line: real part, dashed line: imaginary part).

It is uniquely defined by its values on the rectangle $(\eta, \theta) \in$ $[0,1) \times[0,1 / p)$ and it is a unitary mapping from $L^{2}(\mathbb{R})$ to the Hilbert space $\mathscr{H}^{p} \triangleq L^{2}\left([0,1) \times[0,1 / p) ; \mathbb{C}^{p}\right)$ with inner product

$$
\left\langle\mathscr{Z}_{p} s, \mathscr{Z}_{p} r\right\rangle_{\mathscr{H}^{p}} \triangleq \int_{0}^{1} \int_{0}^{1 / p}\left(\mathscr{Z}_{p} r\right)^{H}(\eta, \theta)\left(\mathscr{Z}_{p} s\right)(\eta, \theta) d \theta d \eta .
$$

Due to their unitarity, Zak transform and PZT preserve the $L^{2}(\mathbb{R})$ inner product $\langle s, r\rangle \triangleq \int_{t} r^{*}(t) s(t) d t$ :

$$
\langle s, r\rangle=\left\langle\mathcal{Z} s, \mathfrak{Z}_{r}\right\rangle_{L^{2}\left(U^{2}\right)}=\left\langle\mathscr{Z}_{p} s, \mathscr{Z}_{p} r\right\rangle_{\mathscr{H}^{p}}
$$

Note that the TF shift covariance properties (11) and (12) also apply to the PZT.

We will further use the following 2D Fourier transform (2D-FT) of $2 \mathrm{D}$ vector sequences $\mathbf{x}_{l, k}$ :

$$
(\mathcal{F} \mathbf{x})(\eta, \theta) \triangleq \sqrt{p} \sum_{l=-\infty}^{\infty} \sum_{k=-\infty}^{\infty} \mathbf{x}_{l, k} e^{-j 2 \pi(l p \theta-k \eta)} .
$$

Like the PZT, $(\mathcal{F} \mathbf{x})(\eta, \theta)$ is uniquely defined by its values on the rectangle $(\eta, \theta) \in[0,1) \times[0,1 / p)$. The $2 \mathrm{D}$-FT is a unitary mapping from the Hilbert space of $2 \mathrm{D}$ sequences in $l^{2}\left(\mathbb{Z}^{2} ; \mathbb{C}^{M}\right), M \in \mathbb{N}$, with inner product $\langle\mathbf{x}, \mathbf{a}\rangle \triangleq \sum_{l, k} \mathbf{a}_{l, k}^{H} \mathbf{x}_{l, k}$ to the Hilbert space $\mathscr{H}^{M}$. The inverse $2 \mathrm{D}$-FT of $\mathbf{A}(\eta, \theta)=$ $(\mathcal{F} \mathbf{a})(\eta, \theta)$ is defined as

$$
\left(\mathcal{F}^{-1} \mathbf{A}\right)_{l, k} \triangleq \sqrt{p} \int_{0}^{1} \int_{0}^{1 / p} \mathbf{A}(\eta, \theta) e^{j 2 \pi(l p \theta-k \eta)} d \theta d \eta .
$$

In practical implementations, PZT and 2D-FT can efficiently be computed using the FFT [24]. 


\subsection{MPMC modulator and demodulator}

We next reformulate the MPMC modulator (4) and the MPMC demodulator (5) in terms of PZTs and 2D-FTs.

Using (12) and (16), the PZT of the MPMC transmit signal in (4) can be calculated as

$$
\begin{aligned}
\left(\mathscr{Z}_{p} s\right)(\eta, \theta) & =\sum_{m=1}^{M} \sum_{l=-\infty}^{\infty} \sum_{k=-\infty}^{\infty}\left(\mathscr{Z}_{p} g_{l, k}^{(m)}\right)(\eta, \theta) a_{l, k}^{(m)} \\
& =\sum_{m=1}^{M}\left(\mathscr{Z}_{p} g^{(m)}\right)(\eta, \theta) \sum_{l=-\infty}^{\infty} \sum_{k=-\infty}^{\infty} e^{-j 2 \pi(l p \theta-k \eta)} a_{l, k}^{(m)} \\
& =\frac{1}{\sqrt{p}} \sum_{m=1}^{M}\left(\mathscr{Z}_{p} g^{(m)}\right)(\eta, \theta)\left(\mathcal{F} a^{(m)}\right)(\eta, \theta) \\
& =\mathbf{M}_{\mathbf{g}}(\eta, \theta)(\mathcal{F} \mathbf{a})(\eta, \theta),
\end{aligned}
$$

where

$$
\mathbf{M}_{\mathbf{g}}(\eta, \theta) \triangleq \frac{1}{\sqrt{p}}\left[\left(\mathscr{Z}_{p} g^{(1)}\right)(\eta, \theta) \cdots\left(\mathscr{Z}_{p} g^{(M)}\right)(\eta, \theta)\right]
$$

denotes the $p \times M$ modulator matrix. Equation (18) constitutes the Zak-Fourier formulation of the MPMC modulator (4) and amounts to a simple matrix-vector multiplication for each $(\eta, \theta)$.

To reformulate the MPMC demodulator (5) in the ZakFourier domain, we use the unitarity (15) and the TF shift covariance (cf. (12)) of the PZT,

$$
\begin{aligned}
x_{l, k}^{(m)} & =\left\langle r, \gamma_{l, k}^{(m)}\right\rangle=\left\langle\mathscr{Z}_{p} r, \mathscr{Z}_{p} \gamma_{l, k}^{(m)}\right\rangle_{\mathcal{H}^{p}} \\
& =\int_{0}^{1} \int_{0}^{1 / p}\left(\mathscr{Z}_{p} \gamma^{(m)}\right)^{H}(\eta, \theta)\left(\mathscr{Z}_{p} r\right)(\eta, \theta) e^{j 2 \pi(l p \theta-k \eta)} d \theta d \eta .
\end{aligned}
$$

Comparing the last expression with (17), we obtain

$$
\begin{aligned}
&\left(\mathcal{F} x^{(m)}\right)(\eta, \theta) \\
& \quad=\frac{1}{\sqrt{p}}\left(\mathscr{Z}_{p} \gamma^{(m)}\right)^{H}(\eta, \theta)\left(\mathscr{Z}_{p} r\right)(\eta, \theta), \quad m=1, \ldots, M,
\end{aligned}
$$

which can again be rewritten as a Zak-Fourier domain matrix-vector multiplication,

$$
(\mathcal{F} \mathbf{x})(\eta, \theta)=\mathbf{D}_{\gamma}(\eta, \theta)\left(\mathscr{Z}_{p} r\right)(\eta, \theta) .
$$

Here, $\mathbf{D}_{\gamma}(\eta, \theta)$ denotes the $M \times p$ demodulator matrix defined as

$$
\mathbf{D}_{\gamma}(\eta, \theta) \triangleq \frac{1}{\sqrt{p}}\left[\left(\mathscr{Z}_{p} \gamma^{(1)}\right)(\eta, \theta) \cdots\left(\mathscr{Z}_{p} \gamma^{(M)}\right)(\eta, \theta)\right]^{H} .
$$

Note that (19) and (23) imply that $\mathbf{M}_{\mathbf{g}}^{H}(\eta, \theta)=\mathbf{D}_{\mathbf{g}}(\eta, \theta)$. Furthermore, for an ideal channel, that is, $r(t)=s(t)$, we have $(\mathcal{F} \mathbf{x})(\eta, \theta)=\mathbf{D}_{\gamma}(\eta, \theta) \mathbf{M}_{\mathbf{g}}(\eta, \theta)(\mathcal{F} \mathbf{a})(\eta, \theta)$. Block diagrams for the Zak-Fourier implementation of MPMC modulator and demodulator are shown in Figure 5.

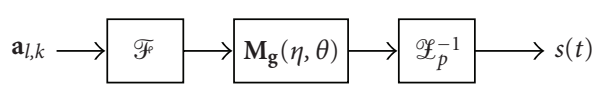

(a)

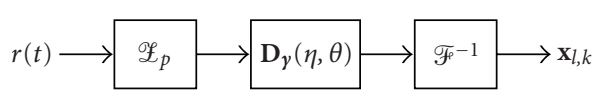

(b)

FIGURE 5: Zak-Fourier domain implementations of (a) MPMC modulator, (b) MPMC demodulator.

\subsection{Rational TF lattice}

The foregoing results for integer TF lattice $(T F=p \in \mathbb{N})$ can straightforwardly be generalized to MPMC systems with rational lattices, $T F=p / q, p, q \in \mathbb{N}$. In particular, an MPMC system with $M \times 1$ transmit/receive multipulses $\mathbf{g}(t), \boldsymbol{\gamma}(t)$ and rational TF lattice $T F=p / q$ can equivalently be viewed as MPMC system with transmit/receive multipulses

$$
\begin{aligned}
& \tilde{\mathbf{g}}(t)=\left[\begin{array}{llll}
\mathbf{g}^{T}(t) & \mathbf{g}^{T}(t-T) & \cdots & \mathbf{g}^{T}(t-(q-1) T)
\end{array}\right]^{T}, \\
& \tilde{\boldsymbol{\gamma}}(t)=\left[\begin{array}{llll}
\boldsymbol{\gamma}^{T}(t) & \boldsymbol{\gamma}^{T}(t-T) & \cdots & \boldsymbol{\gamma}^{T}(t-(q-1) T)
\end{array}\right]^{T},
\end{aligned}
$$

of length $\widetilde{M}=q M$ and integer $\mathrm{TF}$ lattice $\widetilde{T} F=p, \widetilde{T}=q T$. The corresponding transmit/receive symbols are

$$
\begin{aligned}
& \tilde{\mathbf{a}}_{l, k}=\left[\begin{array}{llll}
\mathbf{a}_{q l, k}^{T} & \mathbf{a}_{q l+1, k}^{T} & \cdots & \mathbf{a}_{q l+q-1, k}^{T}
\end{array}\right]^{T}, \\
& \tilde{\mathbf{x}}_{l, k}=\left[\begin{array}{llll}
\mathbf{x}_{q l, k}^{T} & \mathbf{x}_{q l+1, k}^{T} & \cdots & \mathbf{x}_{q l+q-1, k}^{T}
\end{array}\right]^{T} .
\end{aligned}
$$

The previously developed Zak-Fourier formulations of MPMC modulator and demodulator can then be applied to the equivalent MPMC system with integer TF lattice. Note, however, that the size of the matrices and vectors involved (and thus computational complexity) increases with $q$.

\section{4. (BI)ORTHOGONAL MODULATION VIA RIESZ BASES}

This section provides a brief introduction to Riesz bases $[15,25,26]$ and discusses their relevance to linear modulation formats aiming at perfect symbol recovery for ideal channels. The general discussion of this section will be specialized and deepened for MPMC systems in Section 5. We note that Riesz bases involve similar mathematical tools as frames $[15,16,25,26]$ to which they are closely related.

\subsection{Riesz bases}

A sequence $\left\{g_{k}(t)\right\}_{k \in \mathcal{K}}$ of functions in $L^{2}(\mathbb{R})$ with a countable index set $\mathcal{K}$ is called a Riesz basis for its closed linear $\operatorname{span}^{3}$ if and only if there exist two constants $A_{g}, B_{g}$ with

\footnotetext{
${ }^{3}$ For simplicity, the expression "for its closed linear span" will be omitted in the following.
} 
$0<A_{g} \leq B_{g}<\infty$, such that ${ }^{4}$

$$
A_{g} \sum_{k \in \mathcal{K}}\left|a_{k}\right|^{2} \leq\left\|\sum_{k \in \mathcal{K}} a_{k} g_{k}\right\|^{2} \leq B_{g} \sum_{k \in \mathcal{K}}\left|a_{k}\right|^{2}
$$

for any $\left\{a_{k}\right\} \in l^{2}(\mathcal{K})$. The tightest possible bounds $A_{g}, B_{g}$ in (26) are called lower Riesz bound and upper Riesz bound, respectively, and their ratio $B_{g} / A_{g}$ is the condition number of the Riesz basis. If $A_{g}=B_{g}$, then the sequence $\left\{g_{k}(t)\right\}$ is an orthogonal basis (orthonormal if $A_{g}=B_{g}=1$ ). Two sets $\left\{g_{k}(t)\right\},\left\{\gamma_{k}(t)\right\}$ are called biorthogonal if $\left\langle g_{k}, \gamma_{l}\right\rangle=\delta_{k-l}$ for all $k, l \in \mathcal{K}$. If and only if a sequence $\left\{g_{k}(t)\right\}$ is a Riesz basis, then there exists a (not necessarily unique) biorthogonal sequence $\left\{\gamma_{k}(t)\right\}$ which is also a Riesz basis. The Gram operator $g_{g}$ associated to a Riesz basis $\left\{g_{k}(t)\right\}$ is a bounded, positivedefinite (hence also selfadjoint and invertible) linear operator [27] that maps $l^{2}(\mathcal{K})$ to $l^{2}(\mathcal{K})$ according to

$$
\left(g_{g} a\right)_{k} \triangleq \sum_{k^{\prime} \in \mathcal{K}} a_{k^{\prime}}\left\langle g_{k^{\prime}}, g_{k}\right\rangle
$$

For any Riesz basis $\left\{g_{k}(t)\right\}$, an associated modulation (synthesis) operator can be defined as the mapping from $l^{2}(\mathcal{K})$ to $L^{2}(\mathbb{R})$ given by

$$
\left(\mathcal{M}_{g} a\right)(t) \triangleq \sum_{k \in \mathcal{K}} a_{k} g_{k}(t)
$$

Furthermore, a demodulation (analysis) operator $\mathcal{D}_{\gamma}$ mapping $L^{2}(\mathbb{R})$ to $l^{2}(\mathcal{K})$ can be defined for any Riesz basis $\left\{\gamma_{k}(t)\right\}$ as

$$
\left(D_{\gamma} s\right)_{k} \triangleq\left\langle s, \gamma_{k}\right\rangle
$$

Note that any linear modulation scheme can be cast in the forms (28), (29).

If and only if $\left\{g_{k}(t)\right\}$ and $\left\{\gamma_{k}(t)\right\}$ are biorthogonal Riesz bases, then $\mathscr{D}_{\gamma}$ is a left inverse of $\mathcal{M}_{g}$ :

$$
D_{\gamma} \mathcal{M}_{g}=\ell \Longleftrightarrow\left\langle g_{k}, \gamma_{l}\right\rangle=\delta_{k-l}
$$

(for an orthogonal Riesz basis $\mathscr{D}_{g} \mathcal{M}_{g}=A_{g} \ell$ ). In this case, the coefficients $a_{k}$ in (28) can be reobtained from $s(t)=$ $\left(\mathcal{M}_{g} a\right)(t)$ via (29). This corresponds to perfect symbol recovery in a linear modulation scheme with transmit symbols $a_{k}$, transmit pulses $g_{k}(t)$, transmit/receive signal $s(t)$, and receive pulses $\gamma_{k}(t)$.

Note that the adjoint [27] of $\mathscr{D}_{g}$ is given by $\mathscr{D}_{g}^{+}=\mathcal{M}_{g}$, that is, $\left\langle\mathscr{D}_{g} s, a\right\rangle=\left\langle s, \mathcal{M}_{g} a\right\rangle$. Furthermore, the Gram operator can be written as $\mathscr{g}_{g}=\mathscr{D}_{g} \mathcal{M}_{g}$.

\section{2. (Bi)orthogonalization}

We next consider a generalized procedure for computing (bi)orthogonal Riesz bases. Starting from a prescribed Riesz

\footnotetext{
${ }^{4}$ Here, $\|s\|=\sqrt{\langle s, s\rangle}$ denotes the usual $L^{2}(\mathbb{R})$ norm.
}

basis $\left\{g_{k}(t)\right\}$ with associated modulation and Gram operators $\mathcal{M}_{g}$ and $g_{g}$, we define an $\alpha$-parametrized sequence of functions $\left\{g_{k}^{\langle\alpha\rangle}(t)\right\}$ as (cf. [19])

$$
g_{k}^{\langle\alpha\rangle}(t) \triangleq\left(\mathcal{M}_{g} g_{g}^{-1 / 2+\alpha} \delta^{k}\right)(t)
$$

(here, $\left.\delta_{l}^{k}=\delta_{l-k}\right)$. Note that $g_{k}^{\langle 1 / 2\rangle}(t)=g_{k}(t)$.

It can be verified that $\left\{g_{k}^{\langle\alpha\rangle}(t)\right\}$ is also a Riesz basis with associated modulation operator $\mathcal{M}_{g^{\langle\alpha\rangle}}=\mathcal{M}_{g} \mathcal{g}_{g}^{-1 / 2+\alpha}$, demodulation operator $\mathscr{D}_{g^{\langle\alpha\rangle}}=g_{g}^{-1 / 2+\alpha} \mathscr{D}_{g}$, and Gram operator $g_{g}\langle\alpha\rangle=g_{g}^{2 \alpha}$. The associated Riesz bounds are $A_{g^{\langle\alpha\rangle}}=A_{g}^{2 \alpha}$, $B_{g\langle\alpha\rangle}=B_{g}^{2 \alpha}$ for $\alpha \geq 0$ and $A_{g\langle\alpha\rangle}=B_{g}^{2 \alpha}, B_{g\langle\alpha\rangle}=A_{g}^{2 \alpha}$ for $\alpha \leq 0$. Using (30), it is seen that $\left\{g_{k}^{\langle\alpha\rangle}(t)\right\}$ and $\left\{g_{k}^{\langle-\alpha\rangle}(t)\right\}$ are biorthogonal,

$$
\mathcal{D}_{g(-\alpha\rangle} \mathcal{M}_{g}{ }^{\langle\alpha\rangle}=\mathcal{g}_{g}^{-1 / 2-\alpha} \mathcal{D}_{g} \mathcal{M}_{g} \mathcal{g}_{g}^{-1 / 2+\alpha}=\ell
$$

Hence, $\left\{g_{k}^{\langle 0\rangle}(t)\right\}$ is an orthonormal Riesz basis $\left(A_{g}\langle\alpha\rangle=\right.$ $\left.B_{g\langle\alpha\rangle}=1\right)$. Furthermore, $\left\{g_{k}^{\langle-1 / 2\rangle}(t)\right\}$ is the canonical biorthogonal basis for the originally prescribed Riesz basis $\left\{g_{k}(t)\right\}=\left\{g_{k}^{\langle 1 / 2\rangle}(t)\right\}$.

Consider a linear modulation scheme with transmit pulses $\left\{g_{k}^{\langle\alpha\rangle}(t)\right\}$ and receive pulses $\left\{g_{k}^{\langle-\alpha\rangle}(t)\right\}$ derived from a prescribed Riesz basis $\left\{g_{k}(t)\right\}$. There are three important cases.

(i) $\alpha=0$. Here, both transmitter and receiver use the orthogonal pulses $g_{k}^{\langle 0\rangle}(t)=\left(\mathcal{M}_{g} g_{g}^{-1 / 2} \delta^{k}\right)(t)$. For transmission over additive white Gaussian noise (AWGN) channels, this maximizes the SNR after demodulation ("matched filter" [28]).

(ii) $\alpha=1 / 2$. In this case, the modulator uses the prescribed pulses $g_{k}^{\langle 1 / 2\rangle}(t)=g_{k}(t)$ and the demodulator employs the canonical biorthogonal pulses $g_{k}^{\langle-1 / 2\rangle}(t)=$ $\left(\mathcal{M}_{g} g_{g}^{-1} \delta^{k}\right)(t)$.

(iii) $\alpha=-1 / 2$. Now the canonical biorthogonal pulses $g_{k}^{\langle-1 / 2\rangle}(t)$ are used at the transmitter, while the prescribed pulses $g_{k}(t)$ are used at the receiver.

Other choices of $\alpha$ allow to "interpolate" between the above special cases (cf. Section 7).

We finally note that in general, there exist different biorthogonal Riesz bases for a prescribed Riesz basis $\left\{g_{k}(t)\right\}=$ $\left\{g_{k}^{\langle 1 / 2\rangle}(t)\right\}$. This is useful, for example, for optimization purposes. In particular, any biorthogonal Riesz basis can be written as $\left\{g_{k}^{\langle-1 / 2\rangle}(t)+u_{k}(t)\right\}$, where $\left\{g_{k}^{\langle-1 / 2\rangle}(t)\right\}$ is the canonical biorthogonal basis and $\left\{u_{k}(t)\right\}$ is an arbitrary sequence of functions lying in the nullspace of $\mathscr{D}_{g},\left(\mathscr{D}_{g} u_{l}\right)_{k}=0$. This follows from the observation $\left\langle g_{k}, g_{l}^{\langle-1 / 2\rangle}+u_{l}\right\rangle=\delta_{k-l}+\left\langle g_{k}, u_{l}\right\rangle=$ $\delta_{k-l}$ that exploits the fact that $\left\langle g_{k}, u_{l}\right\rangle=\left(\mathscr{D}_{g} u_{l}\right)_{k}^{*}=0$ (cf. (29)).

\section{MULTIPULSE GABOR RIESZ BASES}

The general framework of linear modulation using Riesz bases from the previous section will now be applied to MPMC systems. This leads to the novel notion of multipulse 
Gabor Riesz bases [13] which are closely related to multiwindow Gabor frames [14].

For SPMC systems $(M=1)$, two pulses inducing biorthogonal Gabor Riesz bases on the TF lattice $(l T, k F)$ can be shown to induce dual frames on the adjoint TF lattice $(l / F$, $k / T)$ [29]. This fact has been exploited for the design and analysis of SPMC systems via frame-theoretic tools $[9,19,21]$. Unfortunately, there is no similar duality relation for multipulse Gabor Riesz bases and multiwindow Gabor frames for $M>1$. More precisely, while any two multipulses inducing biorthogonal multipulse Gabor Riesz bases on the TF lattice $(l T, k F)$ also induce dual multiwindow Gabor frames [14] on the adjoint TF lattice, the reverse does not hold in general, that is, there exist dual multiwindow Gabor frames on the adjoint TF lattice that do not correspond to biorthogonal multipulse Gabor Riesz bases on the original TF lattice $(l T, k F)$. Consider, for example, a multiwindow Gabor frame $(M \geq 2)$ with TF lattice $\left(l T^{\prime}, k F^{\prime}\right)$ such that $1 / M<T^{\prime} F^{\prime} \leq M$. Clearly, for the adjoint lattice $(l T, k F)=\left(l / F^{\prime}, k / T^{\prime}\right)$, there is $T F<M$. Hence, the multiwindow Gabor frame cannot induce a multipulse Gabor Riesz basis on the adjoint lattice since this presupposes $T F \geq M$. Nevertheless, we will show in this section that many tools used for multiwindow Gabor frames can be adapted for multipulse Gabor Riesz bases.

\subsection{Definition and properties}

Let us consider sequences of functions $\left\{g_{l, k}^{(m)}(t)\right\}$ with $l, k \in \mathbb{Z}$ and $m \in\{1, \ldots, M\}$, constructed from the $M \times 1$ multipulse $\mathbf{g}(t)=\left[g^{(1)}(t) \cdots g^{(M)}(t)\right]^{T}$ according to

$$
g_{l, k}^{(m)}(t)=g^{(m)}(t-l T) e^{j 2 \pi k F t}
$$

Using the vector notation from Section 2, $\left\{g_{l, k}^{(m)}(t)\right\}$ can more compactly be represented by $\left\{\mathbf{g}_{l, k}(t)\right\}$. We call $\left\{\mathbf{g}_{l, k}(t)\right\}$ a $m u l$ tipulse Gabor Riesz basis if and only if it satisfies (26) (with the single index $k$ replaced by the triple index $(l, k, r) \in$ $\mathbb{Z} \times \mathbb{Z} \times\{1, \ldots, M\})$.

As obvious from (3) and (5), function sets of the form $\left\{\mathbf{g}_{l, k}(t)\right\}$ are the basis of MPMC modulation and demodulation. Restricting to MPMC systems with perfect symbol recovery (i.e., biorthogonality of $\left\{\mathbf{g}_{l, k}(t)\right\}$ and $\left.\left\{\boldsymbol{y}_{l, k}(t)\right\}\right)$ amounts to constraining $\left\{\mathbf{g}_{l, k}(t)\right\}$ to be a multipulse Gabor Riesz basis. ${ }^{5}$ Clearly, the corresponding modulation and demodulation operators are specified by (4) and (5), respectively. However, it is more convenient to analyze multipulse Gabor Riesz bases via the Zak-Fourier framework for MPMC systems introduced in Section 3. Again, for simplicity, we initially restrict ourselves to integer TF lattice, $T F=p \in \mathbb{N}$, and discuss the extension to rational TF lattice later.

In the Zak-Fourier domain, the modulation operator $\mathcal{M}_{\mathrm{g}}$, the demodulation operator $\mathcal{D}_{\mathrm{g}}$, and the Gram operator $g_{\mathrm{g}}=\mathscr{D}_{\mathrm{g}} \mathcal{M}_{\mathrm{g}}$ are represented, respectively, by the $p \times M$

\footnotetext{
${ }^{5}$ Note that practical MPMC systems with $k \in\{0, \ldots, K-1\}$ correspond to setting $a_{l, k}^{(m)}=0$ for $k \notin\{0, \ldots, K-1\}$.
}

modulator matrix $\mathbf{M}_{\mathbf{g}}(\eta, \theta)$ in (18), the $M \times p$ demodulator matrix $\mathbf{D}_{\mathbf{g}}(\eta, \theta)$ in (23), and the $M \times M$ Gram matrix $\mathbf{G}_{\mathbf{g}}(\eta, \theta)=\mathbf{D}_{\mathbf{g}}(\eta, \theta) \mathbf{M}_{\mathbf{g}}(\eta, \theta)$. Note that $\mathbf{D}_{\mathbf{g}}^{H}(\eta, \theta)=\mathbf{M}_{\mathbf{g}}(\eta, \theta)$ and $\mathbf{G}_{\mathbf{g}}(\eta, \theta)=\mathbf{G}_{\mathbf{g}}^{H}(\eta, \theta)$, consistent with $\mathscr{D}_{\mathbf{g}}^{+}=\mathcal{M}_{\mathbf{g}}$ and $\mathcal{g}_{\mathbf{g}}=\mathcal{g}_{\mathbf{g}}^{+}$. The biorthogonality of two multipulse Gabor Riesz bases $\left\{\mathbf{g}_{l, k}(t)\right\},\left\{\boldsymbol{y}_{l, k}(t)\right\}$ amounts to the Zak-Fourier domain relation $\mathbf{D}_{\gamma}(\eta, \theta) \mathbf{M}_{\mathbf{g}}(\eta, \theta)=\mathbf{I}(\mathrm{cf} .(30))$.

Using the shorthand notation $\mathbf{A}(\eta, \theta)=(\mathcal{F} \mathbf{a})(\eta, \theta)$ and the fact that

$$
\begin{aligned}
\left\|\mathbf{M}_{\mathbf{g}} \mathbf{A}\right\|_{\mathscr{H}^{M}}^{2} & =\left\langle\mathbf{M}_{\mathbf{g}} \mathbf{A}, \mathbf{M}_{\mathbf{g}} \mathbf{A}\right\rangle_{\mathscr{H}^{M}}=\left\langle\mathbf{A}, \mathbf{M}_{\mathbf{g}}^{H} \mathbf{M}_{\mathbf{g}} \mathbf{A}\right\rangle_{\mathscr{H}^{M}} \\
& =\left\langle\mathbf{A}, \mathbf{D}_{\mathbf{g}} \mathbf{M}_{\mathbf{g}} \mathbf{A}\right\rangle_{\mathscr{H}^{M}}=\left\langle\mathbf{A}, \mathbf{G}_{\mathbf{g}} \mathbf{A}\right\rangle_{\mathscr{H}^{M}},
\end{aligned}
$$

the Riesz basis definition (26) can be shown to be equivalent to

$$
A_{\mathbf{g}}\|\mathbf{A}\|_{\mathscr{H}^{M}}^{2} \leq\left\langle\mathbf{A}, \mathbf{G}_{\mathbf{g}} \mathbf{A}\right\rangle_{\mathscr{H}^{M}} \leq B_{\mathbf{g}}\|\mathbf{A}\|_{\mathscr{H}^{M}}^{2} .
$$

This inequality is satisfied, if and only if $0<\lambda_{\min } \leq \lambda_{\max }<$ $\infty$, with

$$
\begin{aligned}
& \lambda_{\min } \triangleq \operatorname{essinf}(\eta, \theta) \\
& \lambda_{\max } \triangleq \min _{r \in\{1, \ldots, M\}} \lambda_{r}(\eta, \theta), \\
& \sup _{(\eta, \theta)} \max _{r \in\{1, \ldots, M\}} \lambda_{r}(\eta, \theta),
\end{aligned}
$$

where $\lambda_{r}(\eta, \theta), m=1, \ldots, M$, are the eigenvalues of $\mathbf{G}_{\mathbf{g}}(\eta, \theta)$. As a matter of fact, using similar arguments as in [14], it can be shown that $\lambda_{\min }$ and $\lambda_{\max }$ coincide with the Riesz bounds, that is, $\lambda_{\min }=A_{\mathbf{g}}$ and $\lambda_{\max }=B_{\mathbf{g}}$. Since $\mathbf{G}_{\mathbf{g}}(\eta, \theta)=$ $\mathbf{M}_{\mathbf{g}}^{H}(\eta, \theta) \mathbf{M}_{\mathbf{g}}(\eta, \theta)$ and $\mathbf{M}_{\mathbf{g}}(\eta, \theta)$ is a $p \times M$ matrix, it follows that $\operatorname{rank}\left\{\mathbf{G}_{\mathbf{g}}(\eta, \theta)\right\} \leq p$. Hence, $\mathbf{G}_{\mathbf{g}}(\eta, \theta)$ is singular (i.e., $\left.\lambda_{\min }=0\right)$ if $T F=p<M$. Since this argument extends to rational TF lattice (see below), it follows that the existence of multipulse Gabor Riesz bases requires a TF lattice with $T F \geq M$.

To maximize spectral efficiency in an MPMC system, $T F / M=1$ is desirable. However, for any multipulse Gabor Riesz basis with $T F=M$ and multipulse $\mathbf{g}(t)$, at least one of the inducing pulses has poor temporal or spectral localization, that is, $\operatorname{tg}^{(m)}(t) \notin L^{2}(\mathbb{R})$ or $(d / d t) g^{(m)}(t) \notin L^{2}(\mathbb{R})$ for at least one $m \in\{1, \ldots, M\}$. This follows from the BalianLow-type theorem for multiwindow Gabor frames in [14] by observing that any multipulse Gabor Riesz basis with $T F=M$ is simultaneously a multiwindow Gabor frame. We note, however, that our simulations showed that for redundancies slightly above 1 , the TF localization of MPMC multipulses may be much better than that of SPMC pulses.

\section{2. (Bi)orthogonalization}

The computation of (bi)orthogonal multipulse Gabor Riesz bases according to the methods in Section 4.2 can be performed efficiently in the Zak-Fourier domain.

It can be shown that for multipulse Gabor Riesz bases, the PZT domain equivalent of (31) is ${ }^{6}$

$$
\left(\mathscr{Z}_{p} \mathbf{g}_{l, k}^{\langle\alpha\rangle}\right)(\eta, \theta)=\sqrt{p} \mathbf{M}_{\mathbf{g}}(\eta, \theta) \mathbf{G}_{\mathbf{g}}^{-1 / 2+\alpha}(\eta, \theta) e^{j 2 \pi(l p \eta-k \theta)}
$$

\footnotetext{
${ }^{6}$ We use the notation $\left(\mathscr{Z}_{p} \mathbf{g}\right)(\eta, \theta)=\left[\left(\mathscr{Z}_{p} g^{(1)}\right)(\eta, \theta) \cdots\left(\mathscr{L}_{p} g^{(M)}\right)(\eta, \theta)\right]$
} 
Note that $\mathbf{G}_{\mathbf{g}}^{-1 / 2+\alpha}(\eta, \theta)$ can always be calculated (e.g., via eigenvalue decompositions) since $\mathbf{G}_{\mathbf{g}}(\eta, \theta)$ is positivedefinite almost everywhere (recall that $\lambda_{\min }>0$ ). The TF shift covariance of the PZT (cf. (12)) then implies that $\left\{\mathbf{g}_{l, k}^{\langle\alpha\rangle}(t)\right\}$ is a multipulse Gabor Riesz basis, too (a priori, it is only guaranteed to be a Riesz basis). The inducing multipulse is given by

$$
\left(\mathscr{Z}_{p} \mathbf{g}^{\langle\alpha\rangle}\right)(\eta, \theta)=\sqrt{p} \mathbf{M}_{\mathbf{g}}(\eta, \theta) \mathbf{G}_{\mathbf{g}}^{-1 / 2+\alpha}(\eta, \theta),
$$

that is, the $m$ th pulse of $\mathbf{g}^{\langle\alpha\rangle}(t)$ is obtained as inverse PZT of the $m$ th column of $\mathbf{M}_{\mathbf{g}}(\eta, \theta) \mathbf{G}_{\mathbf{g}}^{-1 / 2+\alpha}(\eta, \theta)$. The corresponding MPMC modulator/demodulator matrices are

$$
\begin{aligned}
\mathbf{M}_{\mathbf{g}}^{\langle\alpha\rangle}(\eta, \theta) & =\mathbf{M}_{\mathbf{g}}(\eta, \theta) \mathbf{G}_{\mathbf{g}}^{-1 / 2+\alpha}(\eta, \theta), \\
\mathbf{D}_{\mathbf{g}}^{\langle\alpha\rangle}(\eta, \theta) & =\left[\mathbf{M}_{\mathbf{g}}^{\langle\alpha\rangle}(\eta, \theta)\right]^{H}=\mathbf{G}_{\mathbf{g}}^{-1 / 2+\alpha}(\eta, \theta) \mathbf{D}_{\mathbf{g}}(\eta, \theta) .
\end{aligned}
$$

The biorthogonality of $\left\{\mathbf{g}_{l, k}^{\langle\alpha\rangle}(t)\right\}$ and $\left\{\mathbf{g}_{l, k}^{\langle-\alpha\rangle}(t)\right\}$ is reflected by the PZT domain relation

$$
\begin{aligned}
& \mathbf{D}_{\mathbf{g}}^{\langle-\alpha\rangle}(\eta, \theta) \mathbf{M}_{\mathbf{g}}^{\langle\alpha\rangle}(\eta, \theta) \\
& \quad=\mathbf{G}_{\mathbf{g}}^{-1 / 2-\alpha}(\eta, \theta) \mathbf{D}_{\mathbf{g}}(\eta, \theta) \mathbf{M}_{\mathbf{g}}(\eta, \theta) \mathbf{G}_{\mathbf{g}}^{-1 / 2+\alpha}(\eta, \theta)=\mathbf{I}
\end{aligned}
$$

We note that $\mathbf{D}_{\mathbf{g}}^{\langle-\alpha\rangle}(\eta, \theta)$ is the Moore-Penrose pseudoinverse of $\mathbf{M}_{\mathbf{g}}^{\langle\alpha\rangle}(\eta, \theta)$.

For a multipulse Gabor Riesz basis with prescribed multipulse $\mathbf{g}^{\langle\alpha\rangle}(t)$, the canonical biorthogonal multipulse is $\mathbf{g}^{\langle-\alpha\rangle}(t)$. Similarly to the general case described in Section 4.2 , the biorthogonal multipulse is not unique, that is, any multipulse of the form $\mathbf{g}^{\langle-\alpha\rangle}(t)+\mathbf{u}(t)$ also induces a biorthogonal multipulse Gabor Riesz basis provided that the elements of $\mathbf{u}(t)$ lie in the nullspace of $\mathcal{D}_{\mathrm{g}}$. The latter can be efficiently computed in the Zak-Fourier domain via singular value decompositions of $\mathbf{D}_{\mathbf{g}}(\eta, \theta)$ for each $(\eta, \theta)$.

Finally, we remark that among all multipulses inducing orthogonal and biorthogonal multipulse Gabor Riesz bases for prescribed $\mathbf{g}(t), \mathbf{g}^{\langle 0\rangle}(t)$ and $\mathbf{g}^{\langle 1 / 2\rangle}(t)$ are closest to $\mathbf{g}(t)$ in $L^{2}$-distance. A proof of this statement is provided in the appendix.

\subsection{Rational TF lattice}

In Section 3.3, we saw that an MPMC system with $M \times 1$ multipulse and rational $\mathrm{TF}$ lattice $T F=p / q, p, q \in \mathbb{N}$ can equivalently be viewed as MPMC system with multipulse $\tilde{\mathbf{g}}(t)=\left[\begin{array}{llll}\mathbf{g}^{T}(t) & \mathbf{g}^{T}(t-T) & \cdots & \mathbf{g}^{T}(t-(q-1) T)\end{array}\right]^{T}$ of length $\widetilde{M}=q M$ and integer TF lattice $\widetilde{T} F=p, \widetilde{T}=q T$. The underlying multipulse Gabor Riesz basis is of course the same in both cases. Calculation of the sequences $\tilde{\mathbf{g}}_{l, k}^{\langle\alpha\rangle}(t)$ for the equivalent system according to (37) yields a multipulse Gabor Riesz basis for the integer lattice. However, it remains to check whether the sequence $\tilde{\mathbf{g}}_{l, k}^{\langle\alpha\rangle}(t)$ also is a multipulse Gabor Riesz basis with respect to the original rational TF lattice, that is, whether $\tilde{\mathbf{g}}^{\langle\alpha\rangle}(t)=$ $\left.\left[\begin{array}{llll}{\left[\mathbf{g}^{\langle\alpha\rangle}(t)\right]^{T}} & {\left[\mathbf{g}^{\langle\alpha\rangle}(t-T)\right]^{T}} & \cdots & {\left[\mathbf{g}^{\langle\alpha\rangle}(t-(q-1) T)\right.}\end{array}\right]^{T}\right]^{T}$.
To this end, we note that due to (11), the demodulator matrix for $\tilde{\mathbf{g}}(t)$ equals

$$
\begin{aligned}
& \mathbf{D}_{\tilde{\mathbf{g}}}(\eta, \theta) \\
& =\left[\begin{array}{llll}
\mathbf{D}_{\mathbf{g}}^{T}(\eta, \theta) & \mathbf{D}_{\mathbf{g}}^{T}\left(\eta-\frac{p}{q}, \theta\right) & \cdots & \mathbf{D}_{\mathbf{g}}^{T}\left(\eta-(q-1) \frac{p}{q}, \theta\right)
\end{array}\right]^{T} .
\end{aligned}
$$

This can be shown to be equivalent to

$$
\Psi \mathbf{D}_{\tilde{\mathbf{g}}}(\eta, \theta)=\Phi \mathbf{D}_{\tilde{\mathbf{g}}}\left(\eta+\frac{p}{q}, \theta\right)
$$

where $\Psi$ is a unitary $q M \times q M$ shift matrix with entries $[\Psi]_{i, j} \triangleq \delta_{(i-j-M \bmod q M)}$ that performs row-shifts by $M$ positions and $\Phi \triangleq \operatorname{diag}\left\{e^{-j 2 \pi p \theta} \mathbf{I}_{M \times M}, \mathbf{I}_{M \times M}, \ldots, \mathbf{I}_{M \times M}\right\}$ is also unitary. To show that $\tilde{\mathbf{g}}_{l, k}^{\langle\alpha\rangle}(t)$ is a multipulse Gabor Riesz basis with respect to the original rational TF lattice, we need to verify that the demodulator matrix $\mathbf{D}_{\widetilde{\mathbf{g}}}^{\langle\alpha\rangle}(\eta, \theta)$ satisfies (42), too:

$$
\begin{aligned}
\Psi \mathbf{D}_{\widetilde{\mathbf{g}}}^{\langle\alpha\rangle}(\eta, \theta)= & \Psi \mathbf{G}_{\tilde{\mathbf{g}}}^{-1 / 2+\alpha}(\eta, \theta) \mathbf{D}_{\widetilde{\mathbf{g}}}(\eta, \theta) \\
& =\Psi\left(\mathbf{D}_{\widetilde{\mathbf{g}}}(\eta, \theta) \mathbf{D}_{\widetilde{\mathbf{g}}}^{H}(\eta, \theta)\right)^{-1 / 2+\alpha} \Psi^{H} \Psi \mathbf{D}_{\tilde{\mathbf{g}}}(\eta, \theta) \\
& =\left(\Psi \mathbf{D}_{\widetilde{\mathbf{g}}}(\eta, \theta) \mathbf{D}_{\widetilde{\mathbf{g}}}^{H}(\eta, \theta) \Psi^{H}\right)^{-1 / 2+\alpha} \Psi \mathbf{D}_{\widetilde{\mathbf{g}}}(\eta, \theta) \\
& =\left(\Phi \mathbf{D}_{\widetilde{\mathbf{g}}}\left(\eta+\frac{p}{q}, \theta\right) \mathbf{D}_{\widetilde{\mathbf{g}}}^{H}\left(\eta+\frac{p}{q}, \theta\right) \Phi^{H}\right)^{-1 / 2+\alpha} \\
& \quad \times \Phi \mathbf{D}_{\widetilde{\mathbf{g}}}\left(\eta+\frac{p}{q}, \theta\right) \\
= & \Phi\left(\mathbf{D}_{\widetilde{\mathbf{g}}}\left(\eta+\frac{p}{q}, \theta\right) \mathbf{D}_{\widetilde{\mathbf{g}}}^{H}\left(\eta+\frac{p}{q}, \theta\right)\right)^{-1 / 2+\alpha} \\
& \quad \times \mathbf{D}_{\tilde{\mathbf{g}}}\left(\eta+\frac{p}{q}, \theta\right) \\
= & \Phi \mathbf{D}_{\widetilde{\mathbf{g}}}^{\langle\alpha\rangle}\left(\eta+\frac{p}{q}, \theta\right) .
\end{aligned}
$$

Here we used (39), $\mathbf{G}_{\widetilde{\mathbf{g}}}(\eta, \theta)=\mathbf{D}_{\widetilde{\mathbf{g}}}(\eta, \theta) \mathbf{D}_{\widetilde{\mathbf{g}}}^{H}(\eta, \theta),(42)$, and the unitarity of $\Psi$ and $\Phi$.

\section{PERFORMANCE ANALYSIS}

While we restricted to ideal channels in the previous sections, we next provide a performance analysis for MPMC transmissions over wireless channels. This will yield benchmark figures for comparing different MPMC (and SPMC) systems.

\subsection{Channel model}

We assume that the received signal equals

$$
r(t)=(\mathbb{H} s)(t)+n(t),
$$

where $n(t)$ is zero-mean additive white Gaussian noise with variance $\sigma_{n}^{2}$ and $\mathbb{U}$ denotes the random time-varying 
multipath channel with input-output relation

$$
\begin{aligned}
(\mathbb{s})(t) & =\int_{\tau} h(t, \tau) s(t-\tau) d \tau+n(t) \\
& =\int_{\tau} \int_{\nu} S_{\mathbb{\sharp}}(\tau, \nu) s(t-\tau) e^{j 2 \pi v t} d \tau d \nu .
\end{aligned}
$$

Here, $h(t, \tau)$ is the channel's time-varying impulse response and $S_{\mathbb{U}}(\tau, \nu) \triangleq \int_{t} h(t, \tau) e^{-j 2 \pi v t} d t$ is the spreading function $[30,31]$ ( $\tau$ and $\nu$ denote time delay and Doppler frequency, resp.). We furthermore assume that the channel satisfies the assumption of wide-sense stationary uncorrelated scattering (WSSUS) [28, 30, 31], which can be formulated as ${ }^{7}$

$$
\mathcal{E}\left\{S_{\mathbb{\sharp}}(\tau, \nu) S_{\mathbb{\sharp}}^{*}\left(\tau^{\prime}, \nu^{\prime}\right)\right\}=C_{\mathbb{\square}}(\tau, \nu) \delta\left(\tau-\tau^{\prime}\right) \delta\left(\nu-\nu^{\prime}\right) .
$$

The second-order channel statistics are completely characterized by the scattering function (delay-Doppler spectrum) $C_{\mathbb{t}}(\tau, \nu)[28,30,31]$. Practical wireless channels are underspread $[28,31]$, that is, the support of $C_{\mathbb{Q}}(\tau, \nu)$ is confined to a small region $\left[-\tau_{\max }, \tau_{\max }\right] \times\left[-\nu_{\max }, \nu_{\max }\right]$ of area $\rho_{\mathbb{U}} \triangleq$ $4 \tau_{\max } \nu_{\max } \ll 1$. We will refer to $\rho_{\mathbb{\uplus}}$ as channel spread.

\subsection{Input-output relation}

For the overall MPMC system including modulator (4), channel (44), and demodulator (5), the input-output relation can be written as

$$
\mathbf{x}_{l, k}=\sum_{l^{\prime}=-\infty}^{\infty} \sum_{k^{\prime}=0}^{K-1} \mathbf{H}_{l, k ; l^{\prime}, k^{\prime}} \mathbf{a}_{l^{\prime}, k^{\prime}}+\mathbf{z}_{l, k},
$$

with the $M \times M$ channel matrices

$$
\mathbf{H}_{l, k ; l^{\prime}, k^{\prime}}=\int_{t} \gamma_{l, k}^{*}(t)\left(\mathbb{M} \mathbf{g}_{l^{\prime}, k^{\prime}}^{T}\right)(t) d t
$$

and the noise vector

$$
\mathbf{z}_{l, k}=\int_{t} \gamma_{l, k}^{*}(t) n(t) d t
$$

Here, the terms with $l^{\prime} \neq l$ and $k^{\prime} \neq k$ correspond to intersymbol and intercarrier interference (ISCI). Furthermore, the off-diagonal elements in $\mathbf{H}_{l, k ; l, k}$ correspond to interpulse interference (IPI), which is specific to MPMC modulation.

Conventional multicarrier designs typically aim at minimizing all interference (ISCI), such that an approximate scalar input-output relation is obtained. For large channel spreads $\rho_{\mathbb{U}}$, that is, for severe delay and Doppler spread, this requires a significant amount of redundancy, which in turn reduces spectral efficiency. The MPMC framework suggests a more general design approach which we call interference shaping. Here, IPI is partially tolerated at the receiver in order to allow further ISCI reduction. Mathematically, this can

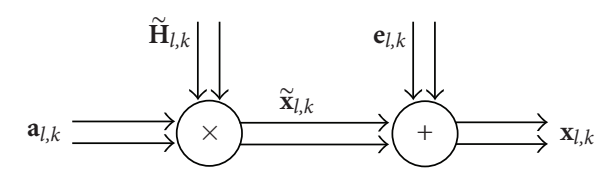

Figure 6: Equivalent vector model for MPMC transmissions.

be expressed via the equivalent input-output relation (see Figure 6)

$$
\mathbf{x}_{l, k}=\tilde{\mathbf{x}}_{l, k}+\mathbf{e}_{l, k}, \quad \text { with } \tilde{\mathbf{x}}_{l, k}=\widetilde{\mathbf{H}}_{l, k} \mathbf{a}_{l, k}
$$

where the channel matrix corresponding to the "desired" receive sequence $\tilde{\mathbf{x}}_{l, k}$ is given by ${ }^{8}$

$$
\widetilde{\mathbf{H}}_{l, k} \triangleq\left[\mathbf{H}_{l, k ; l, k} \odot \mathbf{D}\right] .
$$

The 0/1-valued matrix $\mathbf{D}$ characterizes the intended interference shaping by defining the IPI to be tolerated. All undesired interference (ISCI, undesired IPI) and the noise are subsumed in the interference vector $\mathbf{e}_{l, k}$. If the receiver targets at simple scalar equalization and thus tolerates no IPI, then $\mathbf{D}=\mathbf{I}$. Tolerating all IPI by using more sophisticated matrix equalizers corresponds to $\mathbf{D}=\mathbf{1}$ (the all-one matrix). Note that, according to (50), ISCI and noise are always undesired.

\subsection{Interference analysis}

For the statistical analysis of (50), we assume that the number of subcarriers is infinite (hence, our results provide upper bounds on the interference power for systems with finite number of subcarriers). Furthermore, we assume zeromean i.i.d. transmit symbols $\mathbf{a}_{l, k}$ with correlation matrix $\mathbf{C}_{\mathbf{a}} \triangleq \mathcal{E}\left\{\mathbf{a}_{l, k} \mathbf{a}_{l, k}^{H}\right\}$. Together with the WSSUS assumption, this implies that the actual receive sequence $\mathbf{x}_{l, k}$, the desired sequence $\tilde{\mathbf{x}}_{l, k}$, and the interference sequence $\mathbf{e}_{l, k}$ are i.i.d. sequences with respective correlation matrices $\mathbf{C}_{\mathbf{x}} \triangleq$ $\mathcal{E}\left\{\mathbf{x}_{l, k} \mathbf{x}_{l, k}^{H}\right\}, \mathbf{C}_{\tilde{\mathbf{x}}} \triangleq \mathcal{E}\left\{\widetilde{\mathbf{x}}_{l, k} \widetilde{\mathbf{x}}_{l, k}^{H}\right\}$, and $\mathbf{C}_{\mathbf{e}} \triangleq \mathcal{E}\left\{\mathbf{e}_{l, k} \mathbf{e}_{l, k}^{H}\right\}$. These matrices are related as

$$
\mathbf{C}_{\mathbf{e}}=\mathbf{C}_{\mathbf{x}}-\mathbf{C}_{\mathbf{x}, \tilde{\mathbf{x}}}-\mathbf{C}_{\mathbf{x}, \tilde{\mathbf{x}}}^{H}+\mathbf{C}_{\tilde{\mathbf{x}}}
$$

where $\mathbf{C}_{\mathbf{x}, \tilde{\mathbf{x}}} \triangleq \mathcal{E}\left\{\mathbf{x}_{l, k} \widetilde{\mathbf{x}}_{l, k}^{H}\right\}$. To obtain compact explicit expressions for the above correlation matrices, we introduce the matrix cross-ambiguity function (cf. [32])

$$
\mathbf{A}_{\gamma, \mathbf{g}}(\tau, \nu) \triangleq \int_{t} \gamma(t) \mathbf{g}^{H}(t-\tau) e^{-j 2 \pi v t} d t
$$

of the multipulses $\mathbf{g}(t)$ and $\gamma(t)$, and the periodized scattering function

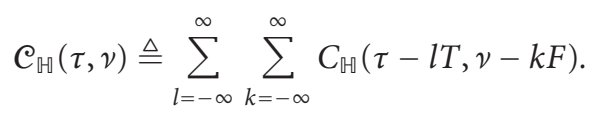

\footnotetext{
$7 \mathscr{E}\{\cdot\}$ denotes expectation (ensemble averaging).
}

\footnotetext{
${ }^{8} \odot$ denotes the Hadamard product.
} 
Note that $\mathcal{C}_{\mathbb{H}}(\tau, \nu)$ depends on the TF lattice parameters $T$ and $F$. With these definitions, it can be shown that

$$
\begin{aligned}
\mathbf{C}_{\tilde{\mathbf{x}}}= & \int_{\tau} \int_{\nu} C_{\mathbb{H}}(\tau, \nu)\left[\mathbf{A}_{\gamma, \mathbf{g}}^{*}(\tau, \nu) \odot \mathbf{D}\right] \\
& \times \mathbf{C}_{\mathbf{a}}\left[\mathbf{A}_{\gamma, \mathbf{g}}(\tau, \nu) \odot \mathbf{D}\right]^{T} d \tau d \nu, \\
\mathbf{C}_{\mathbf{x}}= & \int_{\tau} \int_{\nu} \boldsymbol{C}_{\mathbb{t}}(\tau, \nu) \mathbf{A}_{\gamma, \mathbf{g}}^{*}(\tau, \nu) \mathbf{C}_{\mathbf{a}} \mathbf{A}_{\gamma, \mathbf{g}}^{T}(\tau, \nu) d \tau d \nu \\
& +\sigma_{n}^{2} \int_{t} \gamma^{*}(t) \boldsymbol{\gamma}^{T}(t) d t, \\
\mathbf{C}_{\mathbf{x}, \tilde{\mathbf{x}}}= & \int_{\tau} \int_{\nu} C_{\mathbb{t}}(\tau, \nu) \mathbf{A}_{\gamma, \mathbf{g}}^{*}(\tau, \nu) \mathbf{C}_{\mathbf{a}}\left[\mathbf{A}_{\gamma, \mathbf{g}}(\tau, \nu) \odot \mathbf{D}\right]^{T} d \tau d \nu .
\end{aligned}
$$

These expressions depend on the channel statistics (i.e., scat-

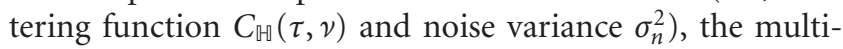
pulses $\mathbf{g}(t)$ and $\boldsymbol{\gamma}(t)$, and on the TF lattice parameters $T, F$. The multipulses and the lattice parameters can be designed to optimize system performance measures like the overall interference power $\mathcal{E}\left\{\mathbf{e}_{l, k}^{H} \mathbf{e}_{l, k}\right\}=\operatorname{tr}\left\{\mathbf{C}_{\mathbf{e}}\right\}$ [17].

\subsection{Spectral efficiency}

Instead of interference power, we aim at using spectral efficiency as performance measure. Based on the vector model (50), the mutual information of $\mathbf{a}_{l, k}$ and $\mathbf{x}_{l, k}$ assuming that $\widetilde{\mathbf{H}}_{l, k}$ is known at the receiver can be calculated as [33]

$$
I_{l, k}=\log _{2} \operatorname{det}\left(\mathbf{I}+\widetilde{\mathbf{H}}_{l, k} \mathbf{C}_{\mathbf{a}} \widetilde{\mathbf{H}}_{l, k}^{H} \mathbf{C}_{\mathbf{e}}^{-1}\right),
$$

where we made the simplifying assumptions that $\mathbf{a}_{l, k}$ and $\mathbf{e}_{l, k}$ are independent and Gaussian. Although $\mathbf{a}_{l, k}$ and $\mathbf{e}_{l, k}$ in practice are correlated, our independence assumption is relevant for receivers that do not exploit these correlations. Furthermore, $\mathbf{a}_{l, k}$ and $\mathbf{e}_{l, k}$ will be approximately Gaussian if linear precoding is used.

Due to the WSSUS assumption, the ergodic mutual information $\mathcal{E}\left\{I_{l, k}\right\}$ is independent of $l, k$. The (ergodic) spectral efficiency in $(\mathrm{bit} / \mathrm{s} / \mathrm{Hz})$ is obtained by normalization with $T F, \zeta \triangleq 1 /(T F) \&\left\{I_{l, k}\right\}$. The expectation involved in $\zeta$ cannot be evaluated explicitly. Since $\log _{2} \operatorname{det}(\cdot)$ is convex, an upper bound is obtained from Jensen's inequality [33],

$$
\begin{aligned}
\zeta & =\frac{1}{T F} \mathscr{E}\left\{\log _{2} \operatorname{det}\left(\mathbf{I}+\widetilde{\mathbf{H}}_{l, k} \mathbf{C}_{\mathbf{a}} \widetilde{\mathbf{H}}_{l, k}^{H} \mathbf{C}_{\mathbf{e}}^{-1}\right)\right\} \leq \zeta_{\max } \\
& \triangleq \frac{1}{T F} \log _{2} \operatorname{det}\left(\mathbf{I}+\mathcal{E}\left\{\widetilde{\mathbf{H}}_{l, k} \mathbf{C}_{\mathbf{a}} \tilde{\mathbf{H}}_{l, k}^{H}\right\} \mathbf{C}_{\mathbf{e}}^{-1}\right) .
\end{aligned}
$$

Since $\widetilde{\mathbf{x}}_{l, k}=\tilde{\mathbf{H}}_{l, k} \mathbf{a}_{l, k}$, we have

$$
\mathcal{E}\left\{\widetilde{\mathbf{H}}_{l, k} \mathbf{C}_{\mathbf{a}} \widetilde{\mathbf{H}}_{l, k}^{H}\right\}=\mathcal{E}\left\{\widetilde{\mathbf{H}}_{l, k} \mathbf{a}_{l, k} \mathbf{a}_{l, k}^{H} \widetilde{\mathbf{H}}_{l, k}^{H}\right\}=\mathbf{C}_{\tilde{\mathbf{x}}},
$$

and thus

$$
\zeta_{\max }=\frac{1}{T F} \log _{2} \operatorname{det}\left(\mathbf{I}+\mathbf{C}_{\widetilde{\mathbf{x}}} \mathbf{C}_{\mathbf{e}}^{-1}\right)
$$

This expression can be easily evaluated for a channel with given scattering function by computing $\mathbf{C}_{\tilde{\mathbf{x}}}, \mathbf{C}_{\mathbf{e}}$ using the results from the previous subsection. In our simulations, we observed that $\zeta_{\max }$ typically is close to $\zeta$. The spectral efficiency measure $\zeta_{\max }$ has the advantage that it allows for fair comparisons of MPMC (SPMC) systems with different lattice parameters $T, F$ and pulse number $M$.

We caution the reader that the TF lattice constants $T$ and $F$ enter (59) twice: explicitly in front of the log and implicitly via the SINR matrix $\mathbf{C}_{\widetilde{\mathbf{x}}} \mathbf{C}_{\mathrm{e}}{ }^{-1}$ (cf. (52) and (55)). While large $T, F$ reduce the pre-log, it simultaneously increases the SINR.

\section{NUMERICAL EXAMPLES}

In this section, we provide design examples for MPMC systems, analyze how MPMC system parameters and channel statistics influence spectral efficiency, and compare MPMC systems with conventional SPMC systems.

\subsection{Simulation setup}

The channel used in the simulations had a flat scattering function $C_{\mathbb{\sharp}}(\tau, \nu)=1 / \rho_{\mathbb{q}}$ for $(\tau, \nu) \in\left[-\tau_{\max }, \tau_{\max }\right] \times\left[-\nu_{\max }\right.$, $\left.\nu_{\max }\right]$ and $C_{\mathbb{\square}}(\tau, \nu)=0$ else. Here, $\tau_{\max }, \nu_{\max }$, and $\rho_{\mathbb{U}}$ are the maximum delay, maximum Doppler, and channel spread, respectively.

The pulse designs presented are based on the methods introduced in Section 5. In all simulations, the prescribed multipulse $\mathbf{g}(t)$ consisted of the first $M=4$ Hermite functions (the SPMC system considered for comparison used the first Hermite function, that is, a Gaussian pulse). This choice was motivated by the fact that these pulses possess the best possible TF localization, that is, their average time-bandwith product $^{9} T_{\mathbf{g}} F_{\mathbf{g}}$ achieves the minimum value of $T_{\mathrm{g}} F_{\mathrm{g}} / M=$ $1 /(4 \pi)$ [32]. Good TF localization is known to be beneficial for reduced ISCI $[9,11,19]$. The scaling of the Hermite functions was matched to the MPMC lattice parameters, $T_{\mathbf{g}} / F_{\mathbf{g}}=T / F$. The design of the MPMC system used $M=4$ and targets at interference shaping with $\mathrm{D}=\mathbf{1}$.

Based on the prescribed Hermite multipulse, biorthogonal multipulses $\mathbf{g}^{\langle\alpha\rangle}(t)$ and $\mathbf{g}^{\langle-\alpha\rangle}(t)$ were calculated according to (38). The resulting MPMC multipulses are depicted in Figures 7(a)-7(c) for $\alpha=1 / 2$ (prescribed Hermite multipulse, $T_{\mathrm{g}} F_{\mathrm{g}} / M=1 /(4 \pi)$ ), $\alpha=0$ (orthogonalized multipulse, $\left.T_{\mathrm{g}} F_{\mathrm{g}} / M=1.13 /(4 \pi)\right)$, and $\alpha=-1 / 2$ (biorthogonalto-Hermite multipulse, $\left.T_{g} F_{g} / M=1.73 /(4 \pi)\right)$. Figures $7(\mathrm{~d})-$ 7 (f) similarly depict the corresponding SPMC pulses which are much poorer localized ${ }^{10}$ (their average time-bandwidth product $T_{g} F_{g}$ equals $1 /(4 \pi), 1.74 /(4 \pi)$, and $6.38 /(4 \pi)$ for $\alpha=1 / 2, \alpha=0$, and $\alpha=-1 / 2$, resp.).

\subsection{TF lattice parameters}

We first investigate the dependence of $\zeta_{\max }$ on the redundancy $T F / M$ and the lattice ratio $T / F$ for an orthogonal MPMC with transmit/receive multipulse $\mathbf{g}^{\langle 0\rangle}(t)$.

\footnotetext{
${ }^{9} T_{\mathbf{g}}^{2} \triangleq \int_{t} t^{2} \mathbf{g}^{H}(t) \mathbf{g}(t) d t / \int_{t} \mathbf{g}^{H}(t) \mathbf{g}(t) d t, F_{\mathbf{g}}^{2} \triangleq \int_{f} f^{2} \mathbf{G}^{H}(f) \mathbf{G}(f) d f /$ $\int_{f} \mathbf{G}^{H}(f) \mathbf{G}(f) d f$, where $\mathbf{G}(f)$ denotes the Fourier transform of $\mathbf{g}(t)$.
}

${ }^{10}$ For proper comparison of Figures $7(\mathrm{a})-7$ (c) and 7 (d) -7 (f), it should be kept in mind that the symbol period $T$ and subcarrier spacing $F$ of the MPMC system are twice as large as that of the SPMC system. 


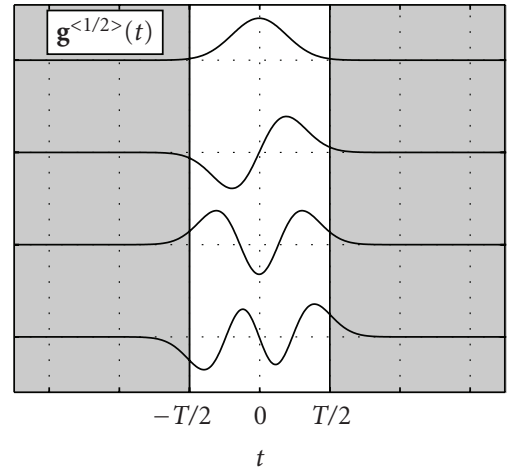

(a)

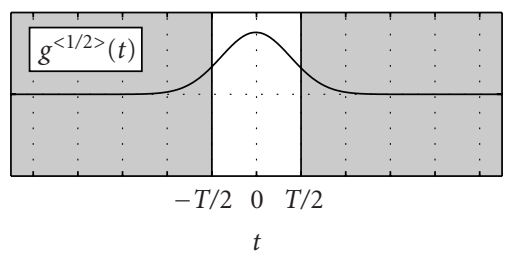

(d)

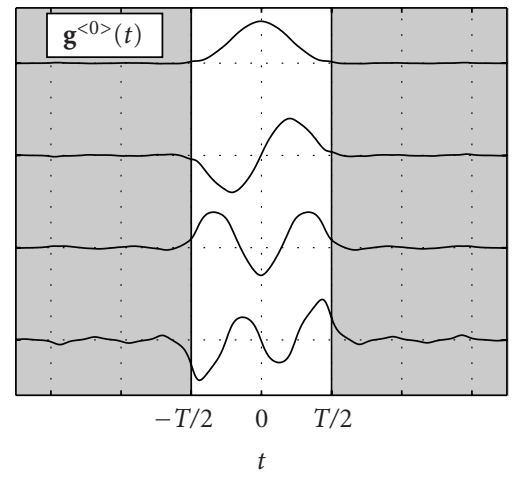

(b)

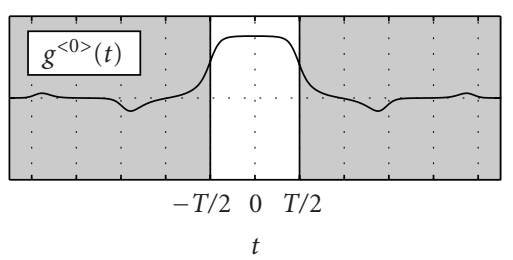

(e)

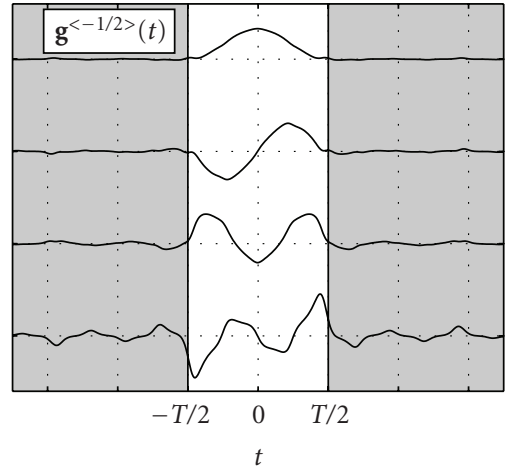

(c)

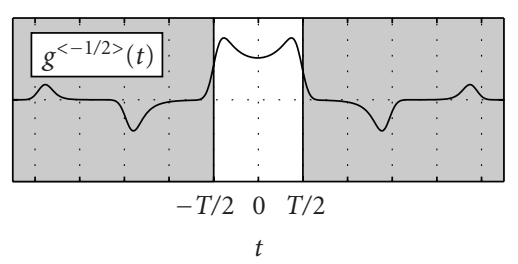

(f)

Figure 7: MPMC multipulse for (a) $\alpha=1 / 2$, (b) $\alpha=0$, (c) $\alpha=-1 / 2$, and SPMC pulse for (d) $\alpha=1 / 2$, (e) $\alpha=0$, (f) $\alpha=-1 / 2$.

In Figure 8(a), $\zeta_{\max }$ is plotted versus $T F / M \in[1.0125$, 1.2] for different SNRs and channel spreads. In all cases, the lattice ratio was $T / F=\tau_{\max } / \nu_{\max }$. As expected, large redundancy, low SNR, and large channel spreads degrade spectral efficiency. The degradation due to channel dispersion is particularly pronounced for large SNR. The optimum redundancy that maximizes $\zeta_{\max }$ (marked with $\times$ ) is seen to range from $\approx 1.0125$ (for low SNR or low channel spread) to $\approx 1.0375$ (for large SNR and large channel spread).

Spectral efficiency versus the normalized lattice ratio $(T / F) /\left(\tau_{\max } / \nu_{\max }\right)$ with fixed redundancy $T F / M=17 / 16=$ 1.0625 is shown in Figure 8(b), again for various SNRs and channel spreads. The simulation confirms that for all channel spreads and SNRs, the optimum TF lattice ratio equals $T / F=\tau_{\max } / \nu_{\max }$, although the dependence of $\zeta_{\max }$ on $T / F$ is weak for low $\rho_{\sharp}$. The same result, not shown, was obtained for the SPMC system using an orthogonalized Gaussian.

\subsection{Biorthogonalization parameter}

Next, we analyze how spectral efficiency depends on the parameter $\alpha$ used to calculate the biorthogonal transmit and receive multipulses. Here, $T F / M=1.0625, T / F=\tau_{\max } / \nu_{\max }$, and $\rho_{\boxplus}=0.0083$.

The spectral efficiency $\zeta_{\max }$ for the MPMC system and the SPMC system is shown in Figures 9(a) and 9(b), respectively, for several SNR values. For low SNR, the spectral efficiency of the MPMC and the SPMC system is maximized by choosing $\alpha=0$, that is, orthogonal pulses amounting to matched filtering. For larger SNRs (i.e., interference-limited situations), however, the optimum $\alpha$ for the MPMC system tends to $-1 / 2$, corresponding to a Hermite multipulse at the receiver and its biorthogonal multipulse at the transmitter.
This is intuitive since the localization of the transmit pulses is destroyed by the channel anyway, and hence the perfect localization of the Hermite multipulse is best exploited at the receiver. With the SPMC system, the optimum $\alpha$ for large SNR is slightly above 0 . However, $\alpha=0$ is close to optimum for all SNRs.

\subsection{System comparison}

Finally, we compare the spectral efficiencies of the MPMC system with orthogonalized Hermite multipulse, the SPMC system with orthogonalized Gaussian, and a conventional CP-OFDM system. All systems had redundancy $T F / M=$ $17 / 16$ and lattice ratio $T / F=\tau_{\max } / \nu_{\max }$. The SNR was $30 \mathrm{~dB}$ and maximum delay and Doppler were varied in a range such that $\sqrt{F / T} \tau_{\max } \in[0,0.1]$, and $\sqrt{T / F} v_{\max } \in[0,0.1]$.

We note that pulse optimization procedures exist both for SPMC systems [11, 34] and MPMC systems [17]. Furthermore, $[18,34,35]$ discuss the practically relevant design and optimization of finite-duration pulses. System comparisons using optimized pulses are, however, beyond the scope of this paper.

Figure 10 shows that for all channel parameters, the MPMC system outperforms the SPMC system. In almost all cases, the MPMC and SPMC systems have larger $\zeta_{\max }$ than the CP-OFDM system. The latter is advantageous only for small Doppler and for delays below the CP duration. For

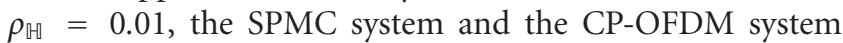
loose $0.9 \mathrm{bit} / \mathrm{s} / \mathrm{Hz}$ and $1.7 \mathrm{bit} / \mathrm{s} / \mathrm{Hz}$ in spectral efficiency compared to the MPMC system. We finally note that the MPMC system is much more robust to larger channel spreads, that is, the spectral efficiency at $\rho_{\nVdash}=0.01$ is only $1.2 \mathrm{bit} / \mathrm{s} / \mathrm{Hz}$ less than at $\rho_{\mathbb{H}}=0$. The corresponding decrease in spectral 


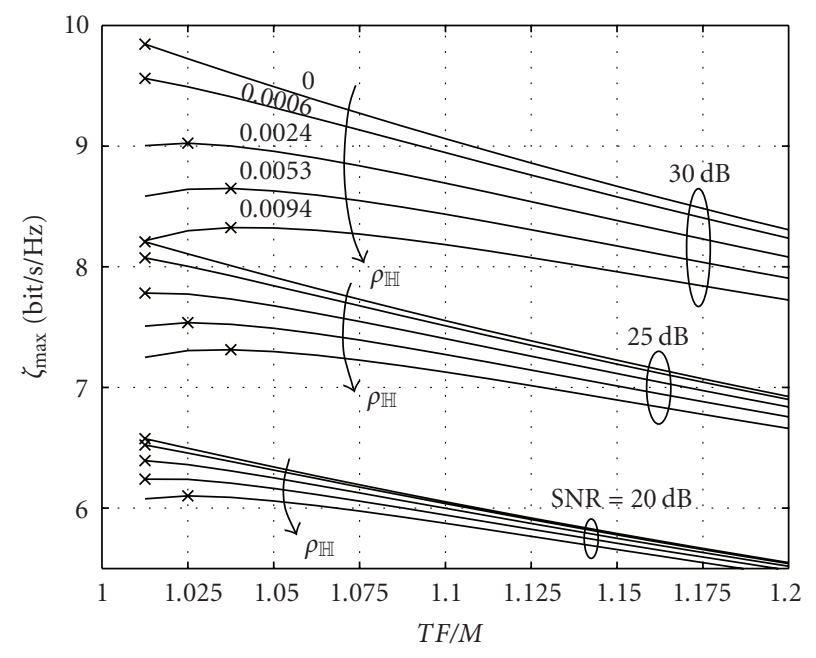

(a)

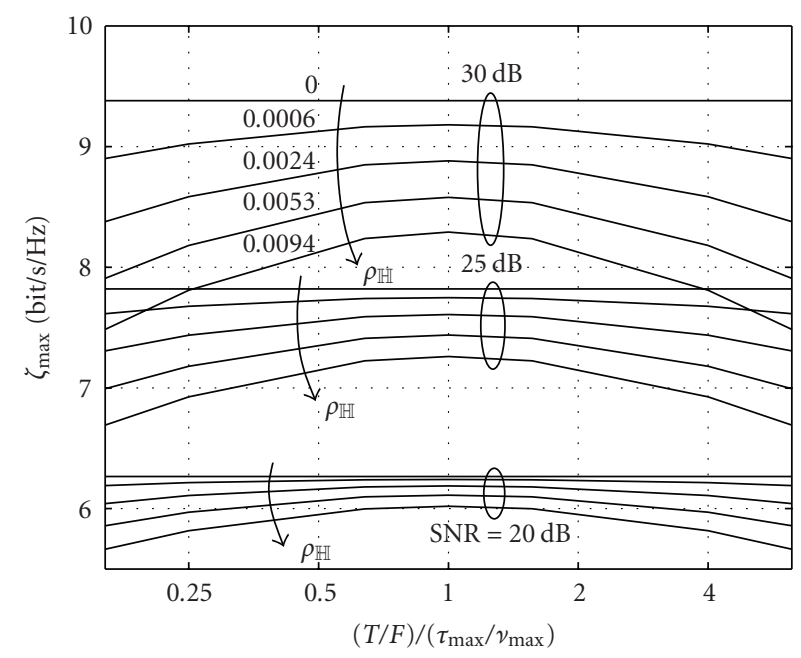

(b)

FIGURE 8: Spectral efficiency of MPMC system with $M=4$ orthogonalized Hermite pulses (a) versus redundancy $T F / M$ and (b) versus normalized lattice ratio $(T / F) /\left(\tau_{\max } / \nu_{\max }\right)$.

efficiency for the SPMC system and the CP-OFDM system is $2.1 \mathrm{bit} / \mathrm{s} / \mathrm{Hz}$ and $2.9 \mathrm{bit} / \mathrm{s} / \mathrm{Hz}$, respectively.

\section{CONCLUSIONS}

We introduced and analyzed multipulse multicarrier (MPMC) modulation, a generalization of traditional multicarrier modulation that uses multiple transmit and receive pulses in parallel. It was shown that multipulse Gabor Riesz bases constitute the theoretical foundation of MPMC systems. Both multipulse Gabor Riesz bases and MPMC systems can be efficiently analyzed and designed in the Zak-Fourier domain via tools that have previously successfully been used in the context of multiwindow Gabor frames. The construction of biorthogonal multipulse Gabor Riesz bases received special attention as it allows to design MPMC systems with perfect symbol recovery. Numerical

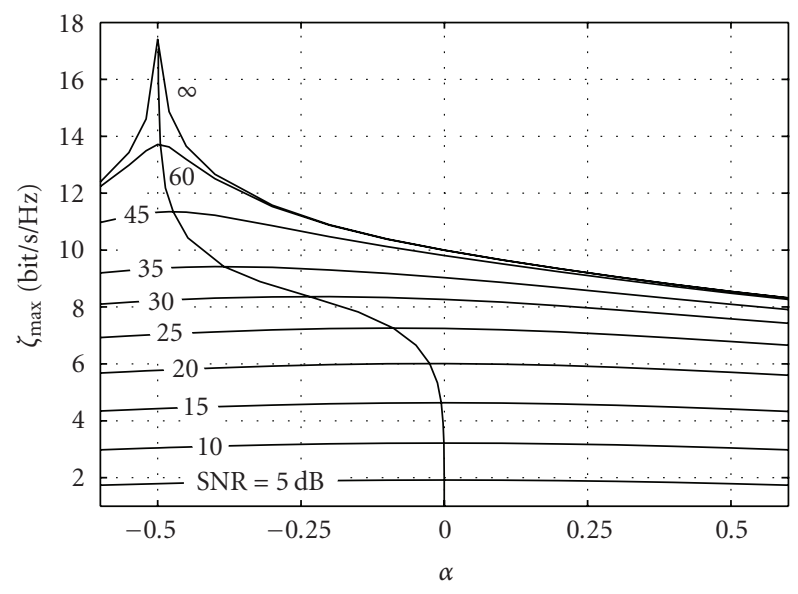

(a)

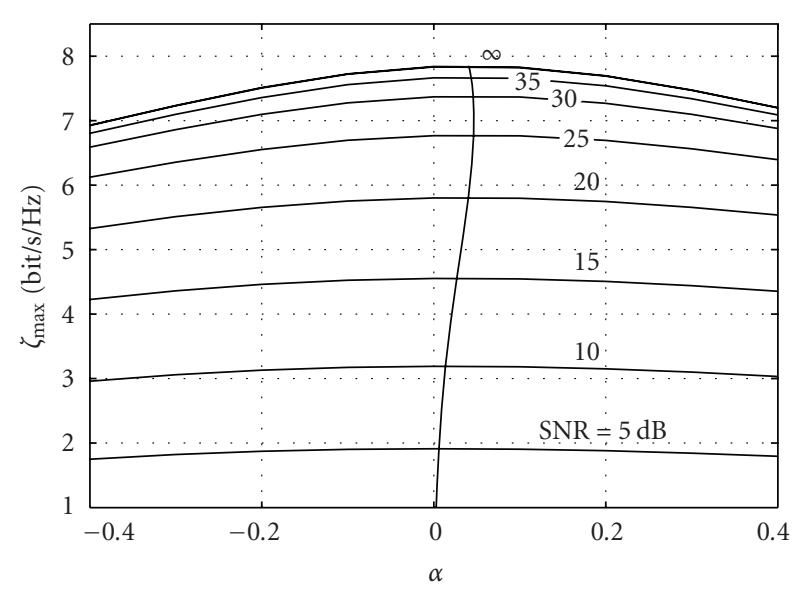

(b)

FIGURE 9: Spectral efficiency $\zeta_{\max }$ versus $\alpha$ obtained with (a) the MPMC system and (b) the SPMC system for different SNRs $\left(T F / M=1.0625\right.$ and $\left.\rho_{\mathbb{H}}=0.0083\right)$. The vertical trajectories indicate the $\alpha$ values that maximize $\zeta_{\max }$ at the various SNRs.

examples were presented that illustrated the MPMC pulse design and showed that MPMC systems offer significant spectral efficiency gains compared to traditional single-pulse multicarrier systems. These gains are partly due to the fact that the MPMC design tolerates a certain amount of "interpulse" interference. This comes at the price of slightly increased receiver complexity (matrix equalizers, etc.). We note that an efficient implementation of MPMC systems augmented by precoding and channel estimation is presented in [18]. Methods for multipulse optimization have been proposed in [17].

\section{APPENDIX}

In this appendix, we show that for a given multipulse $\mathbf{g}(t)=$ $\mathbf{g}^{\langle 1 / 2\rangle}(t)$, the multipulses $\mathbf{g}^{\langle 0\rangle}(t)$ and $\mathbf{g}^{\langle-1 / 2\rangle}(t)$ are the closest to $\mathbf{g}(t)$ among all multipulses inducing (bi)orthogonal multipulse Gabor Riesz bases. A similar proof for canonical tight Gabor frames was given in [36]. The underlying (squared) 


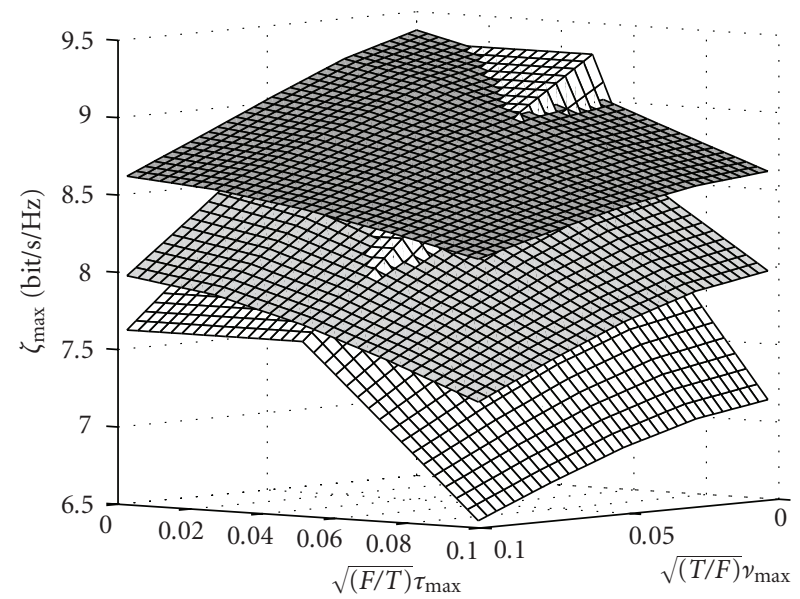

FIGURE 10: Spectral efficiency versus (normalized) maximum delay and Doppler at SNR $=30 \mathrm{~dB}$ for the MPMC system (dark gray), the SPMC system (light gray), and the CP-OFDM system (white).

distance of a multipulse $\boldsymbol{\gamma}(t)$ from the prescribed multipulse $\mathbf{g}(t)$ is

$$
\begin{aligned}
d^{2}(\mathbf{g}, \boldsymbol{\gamma}) & \triangleq \sum_{m=1}^{M} \int_{t}\left|g^{(m)}(t)-\gamma^{(m)}(t)\right|^{2} d t=\sum_{m=1}^{M}\left\|g^{(m)}-\gamma^{(m)}\right\|^{2} \\
& =\sum_{m=1}^{M}\left[\left\|g^{(m)}\right\|^{2}-2 \operatorname{Re}\left\{\left\langle g^{(m)}, \gamma^{(m)}\right\rangle\right\}+\left\|\gamma^{(m)}\right\|^{2}\right] .
\end{aligned}
$$

Consider first the case where $\gamma(t)$ is required to induce an orthonormal multipulse Gabor Riesz basis. This implies that $\sum_{m=1}^{M}\left\|\gamma^{(m)}\right\|_{2}^{2}=M$. Since $\sum_{m=1}^{M}\left\|g^{(m)}\right\|_{2}^{2}$ is fixed, minimization of (A.1) is equivalent to maximization of $\sum_{m=1}^{M} \operatorname{Re}\left\{\left\langle g^{(m)}, \gamma^{(m)}\right\rangle\right\}$. The unitarity of the PZT yields

$$
\begin{aligned}
\sum_{m=1}^{M}\left\langle g^{(m)}, \gamma^{(m)}\right\rangle & =\sum_{m=1}^{M}\left\langle\mathscr{Z}_{g^{(m)}}, \mathscr{Z}_{\mathbf{\gamma}}^{(m)}\right\rangle \\
& =\int_{0}^{1} \int_{0}^{1 / p} \operatorname{tr}\left\{\mathbf{M}_{\mathbf{g}}^{H}(\eta, \theta) \mathbf{M}_{\gamma}(\eta, \theta)\right\} d \theta d \eta
\end{aligned}
$$

The orthonormality constraint for $\left\{\gamma_{l, k}(t)\right\}$ is equivalent to $\mathbf{M}_{\gamma}(\eta, \theta) \in \mathcal{O} \triangleq\left\{\mathbf{M}_{\gamma}(\eta, \theta): \mathbf{M}_{\gamma}^{H}(\eta, \theta) \mathbf{M}_{\gamma}(\eta, \theta)=\mathbf{I}\right\}$. It can be shown that [37]

$$
\begin{aligned}
\max _{\mathbf{M}_{\gamma}(\eta, \theta) \in \mathcal{O}} \operatorname{tr}\left\{\mathbf{M}_{\mathbf{g}}^{H}(\eta, \theta) \mathbf{M}_{\gamma}(\eta, \theta)\right\} \\
=\operatorname{tr}\left\{\left(\mathbf{M}_{\mathbf{g}}^{H}(\eta, \theta) \mathbf{M}_{\mathbf{g}}(\eta, \theta)\right)^{1 / 2}\right\},
\end{aligned}
$$

where the (real-valued) maximum is achieved with

$$
\begin{aligned}
\mathbf{M}_{\gamma}(\eta, \theta) & =\mathbf{M}_{\mathbf{g}}^{\langle 0\rangle}(\eta, \theta) \\
& =\mathbf{M}_{\mathbf{g}}(\eta, \theta)\left(\mathbf{M}_{\mathbf{g}}^{H}(\eta, \theta) \mathbf{M}_{\mathbf{g}}(\eta, \theta)\right)^{-1 / 2}
\end{aligned}
$$

This proves that $\gamma(t)=\mathbf{g}^{\langle 0\rangle}(t)$ maximizes $\operatorname{Re}\left\{\sum_{m=1}^{M}\left\langle g^{(m)}\right.\right.$, $\left.\left.\gamma^{(m)}\right\rangle\right\}$, and hence minimizes (A.1).

Next consider the case where $\boldsymbol{\gamma}(t)$ is constrained to induce a multipulse Gabor Riesz basis biorthogonal to that induced by the fixed multipulse $\mathbf{g}(t)$. This implies that $\sum_{m=1}^{M}\left\langle g^{(m)}, \gamma^{(m)}\right\rangle=M$, such that minimization of (A.1) is equivalent to minimization of

$$
\begin{aligned}
\sum_{m=1}^{M}\left\|\gamma^{(m)}\right\|^{2} & =\sum_{m=1}^{M}\left\langle\mathscr{C} \gamma^{(m)}, \mathfrak{Z} \gamma^{(m)}\right\rangle \\
& =\int_{0}^{1} \int_{0}^{1 / p} \operatorname{tr}\left\{\mathbf{M}_{\gamma}^{H}(\eta, \theta) \mathbf{M}_{\gamma}(\eta, \theta)\right\} d \theta d \eta .
\end{aligned}
$$

The biorthogonality constraint for $\left\{\boldsymbol{y}_{l, k}(t)\right\}$ is equivalent to $\mathbf{M}_{\gamma}(\eta, \theta) \in \mathscr{B} \triangleq\left\{\mathbf{M}_{\gamma}(\eta, \theta): \mathbf{M}_{\mathbf{g}}^{H}(\eta, \theta) \mathbf{M}_{\gamma}(\eta, \theta)=\mathbf{I}\right\}$. It can be shown that [38]

$$
\begin{aligned}
\min _{\mathbf{M}_{y}(\eta, \theta) \in \mathcal{B}} \operatorname{tr}\left\{\mathbf{M}_{\gamma}^{H}(\eta, \theta) \mathbf{M}_{y}(\eta, \theta)\right\} \\
=\operatorname{tr}\left\{\left(\mathbf{M}_{\mathbf{g}}^{H}(\eta, \theta) \mathbf{M}_{\mathbf{g}}(\eta, \theta)\right)^{-1}\right\},
\end{aligned}
$$

where the minimum is achieved with

$$
\begin{aligned}
\mathbf{M}_{\gamma}(\eta, \theta) & =\mathbf{M}_{\mathbf{g}}^{\langle-1 / 2\rangle}(\eta, \theta) \\
& =\mathbf{M}_{\mathbf{g}}(\eta, \theta)\left(\mathbf{M}_{\mathbf{g}}^{H}(\eta, \theta) \mathbf{M}_{\mathbf{g}}(\eta, \theta)\right)^{-1} .
\end{aligned}
$$

Thus, among all biorthogonal multipulses $\gamma(t)$, the canonical biorthogonal multipulse $\gamma(t)=\mathbf{g}^{\langle-1 / 2\rangle}(t)$ minimizes $\sum_{m=1}^{M}\left\|\gamma^{(m)}\right\|^{2}$ and therefore $d^{2}(\mathbf{g}, \boldsymbol{\gamma})$.

\section{ACKNOWLEDGMENT}

This work was funded by FWF Grants P15156 and J2302.

\section{REFERENCES}

[1] R. W. Chang, "Synthesis of band-limited orthogonal signals for multi-channel data transmission," Bell System Technical Journal, vol. 45, no. 10, pp. 1775-1796, 1966.

[2] J. A. C. Bingham, "Multicarrier modulation for data transmission: an idea whose time has come," IEEE Communications Magazine, vol. 28, no. 5, pp. 5-14, 1990.

[3] B. Le Floch, M. Alard, and C. Berrou, "Coded orthogonal frequency division multiplex," Proceedings of the IEEE, vol. 83, no. 6, pp. 982-996, 1995.

[4] S. B. Weinstein and P. M. Ebert, "Data transmission by frequency division multiplexing using the discrete Fourier transform," IEEE Transactions on Communications, vol. 19, no. 5, part 1, pp. 628-634, 1971.

[5] L. Cimini Jr., "Analysis and simulation of a digital mobile channel using orthogonal frequency division multiplexing," IEEE Transactions on Communications, vol. 33, no. 7, pp. 665675, 1985.

[6] A. Peled and A. Ruiz, "Frequency domain data transmission using reduced computational complexity algorithms," in Proceedings of IEEE International Conference on Acoustics, Speech and Signal Processing (ICASSP '80), vol. 5, pp. 964-967, Denver, Colo, USA, April 1980. 
[7] P. Smulders, "Exploiting the $60 \mathrm{GHz}$ band for local wireless multimedia access: prospects and future directions," IEEE Communications Magazine, vol. 40, no. 1, pp. 140-147, 2002.

[8] R. Haas and J.-C. Belfiore, "A time-frequency well-localized pulse for multiple carrier transmission," Wireless Personal Communications, vol. 5, no. 1, pp. 1-18, 1997.

[9] H. Bölcskei, "Efficient design of pulse-shaping filters for OFDM systems," in Wavelet Applications in Signal and Image Processing VII, vol. 3813 of Proceedings of SPIE, pp. 625-636, Denver, Colo, USA, July 1999.

[10] W. Kozek and A. F. Molisch, "Nonorthogonal pulseshapes for multicarrier communications in doubly dispersive channels," IEEE Journal on Selected Areas in Communications, vol. 16, no. 8, pp. 1579-1589, 1998.

[11] D. Schafhuber, G. Matz, and F. Hlawatsch, "Pulse-shaping OFDM/BFDM systems for time-varying channels: ISI/ICI analysis, optimal pulse design, and efficient implementation," in Proceedings of 13th IEEE International Symposium on Personal, Indoor and Mobile Radio Communications (PIMRC '02), vol. 3, pp. 1012-1016, Lisbon, Portugal, September 2002.

[12] C. Siclet, P. Siohan, and D. Pinchon, "Oversampled orthogonal and biorthogonal multicarrier modulations with perfect reconstruction," in Proceedings of IEEE 14th International Conference on Digital Signal Processing (DSP '02), vol. 2, pp. 647650, Santorini, Greece, July 2002.

[13] M. M. Hartmann, G. Matz, and D. Schafhuber, "Theory and design of multipulse multicarrier systems for wireless communications," in Proceedings of 37th IEEE Asilomar Conference on Signals, Systems and Computers (ACSSC '03), vol. 1, pp. 492496, Pacific Grove, Calif, USA, November 2003.

[14] M. Zibulski and Y. Y. Zeevi, "Analysis of multiwindow Gabortype schemes by frame methods," Applied and Computational Harmonic Analysis, vol. 4, no. 2, pp. 188-221, 1997.

[15] K. Gröchenig, Foundations of Time-Frequency Analysis, Birkhäuser, Boston, Mass, USA, 2001.

[16] H. G. Feichtinger and T. Strohmer, Eds., Gabor Analysis and Algorithms: Theory and Applications, Birkhäuser, Boston, Mass, USA, 1998.

[17] M. M. Hartmann, G. Matz, and D. Schafhuber, "Multipulse multicarrier communications over time-varying fading channels: performance analysis and system optimization," in Proceedings of IEEE International Conference on Acoustics, Speech and Signal Processing (ICASSP '04), vol. 3, pp. 805-808, Montreal, Quebec, Canada, May 2004.

[18] M. M. Hartmann, G. Matz, and D. Schafhuber, "Transceiver design for precoded multipulse multicarrier packet transmissions over time-varying fading channels," in Proceedings of IEEE Global Telecommunications Conference (GLOBECOM '04), vol. 4, pp. 2409-2413, Dallas, Tex, USA, NovemberDecember 2004.

[19] T. Strohmer and S. Beaver, "Optimal OFDM design for timefrequency dispersive channels," IEEE Transactions on Communications, vol. 51, no. 7, pp. 1111-1122, 2003.

[20] B. Hirosaki, "An orthogonally multiplexed QAM system using the discrete Fourier transform," IEEE Transactions on Communications, vol. 29, no. 7, pp. 982-989, 1981.

[21] H. Bölcskei, P. Duhamel, and R. Hleiss, "Design of pulse shaping OFDM/OQAM systems for high data-rate transmission over wireless channels," in Proceedings of IEEE International Conference on Communications (ICC '99), vol. 1, pp. 559-564, Vancouver, British Columbia, Canada, June 1999.

[22] P. Siohan, C. Siclet, and N. Lacaille, "Analysis and design of OFDM/OQAM systems based on filterbank theory," IEEE Transactions on Signal Processing, vol. 50, no. 5, pp. 1170-1183, 2002.
[23] S. Hara and R. Prasad, "Overview of multicarrier CDMA," IEEE Communications Magazine, vol. 35, no. 12, pp. 126-133, 1997.

[24] M. Zibulski and Y. Y. Zeevi, "Discrete multiwindow Gabortype transforms," IEEE Transactions on Signal Processing, vol. 45, no. 6, pp. 1428-1442, 1997.

[25] O. Christensen, An Introduction to Frames and Riesz Bases, Birkhäuser, Boston, Mass, USA, 2003.

[26] R. M. Young, An Introduction to Nonharmonic Fourier Series, Academic Press, New York, NY, USA, 1980.

[27] A. W. Naylor and G. R. Sell, Linear Operator Theory in Engineering and Science, Springer, New York, NY, USA, 2nd edition, 1982.

[28] J. G. Proakis, Digital Communications, McGraw-Hill, New York, NY, USA, 3rd edition, 1995.

[29] A. J. E. M. Janssen, "Duality and biorthogonality for WeylHeisenberg frames," Journal of Fourier Analysis and Applications, vol. 1, no. 4, pp. 403-436, 1995.

[30] P. A. Bello, "Characterization of randomly time-variant linear channels," IEEE Transactions on Communications Systems, vol. 11, no. 4, pp. 360-393, 1963.

[31] G. Matz and F. Hlawatsch, "Time-frequency characterization of random time-varying channels," in Time-Frequency Signal Analysis and Processing: A Comprehensive Reference, B. Boashash, Ed., chapter 9.5, pp. 410-419, Elsevier, Oxford, UK, 2003.

[32] F. Hlawatsch, Time-Frequency Analysis and Synthesis of Linear Signal Spaces: Time-Frequency Filters, Signal Detection and Estimation, and Range-Doppler Estimation, Kluwer Academic, Boston, Mass, USA, 1998.

[33] T. M. Cover and J. A. Thomas, Elements of Information Theory, John Wiley \& Sons, New York, NY, USA, 1991.

[34] D. Pinchon, P. Siohan, and C. Siclet, "Design techniques for orthogonal modulated filterbanks based on a compact representation," IEEE Transactions on Signal Processing, vol. 52, no. 6, pp. 1682-1692, 2004.

[35] H. Bölcskei and A. J. E. M. Janssen, "Gabor frames, unimodularity, and window decay," Journal of Fourier Analysis and Applications, vol. 6, no. 3, pp. 255-276, 2000.

[36] A. J. E. M. Janssen and T. Strohmer, "Characterization and computation of canonical tight windows for Gabor frames," Journal of Fourier Analysis and Applications, vol. 8, no. 1, pp. $1-28,2002$.

[37] R. A. Horn and C. R. Johnson, Topics in Matrix Analysis, Cambridge University Press, Cambridge, UK, 1994.

[38] G. H. Golub and C. F. Van Loan, Matrix Computations, Johns Hopkins University Press, Baltimore, Md, USA, 2nd edition, 1989.

Manfred M. Hartmann received the Dipl.Ing. degree in electrical engineering from Vienna University of Technology, Austria, in April 2003. From May 2003 till December 2004, he has been with the Institute of Communications and Radio Frequency Engineering, Vienna University of Technology, working as a Research Assistant within the project "Advanced Multicarrier Systems for Wireless Communications."

Since March 2005, he has been with ARC Seibersdorf Research $\mathrm{GmbH}$, Vienna. His research interests include statistical signal processing, wireless communications, and biosignal processing. 
Gerald Matz received the Dipl.-Ing. and Dr. Techn. degrees in electrical engineering and the Habilitation degree for communication systems from Vienna University of Technology, Austria, in December 1994, December 2000, and September 2004, respectively. Since January 1995, he has been with the Institute of Communications and Radio Frequency Engineering, Vienna University of Technology, where he currently holds a tenured Associate Professor position. From March 2004 to February 2005, he was an Erwin Schrödinger Fellow with the Laboratoire des Signaux et Systèmes, Ecole Supérieure d'Electricité (France). Professor Matz has directed or actively participated in several research projects funded by the Austrian Science Fund (FWF) and by the European Union. He has published some 70 technical articles in international journals, conference proceedings, and edited books. He serves as an Associate Editor of the IEEE Signal Processing Letters, was the Technical Program Cochair of EUSIPCO 2004, and Member of the Program Committee of several international conferences. His research interests include wireless communications, statistical signal processing, and time-frequency signal processing. He is a Member of IEEE and ÖVE.

Dieter Schafhuber received the Dipl.-Ing. and Dr. Techn. degrees in electrical engineering from Vienna University of Technology, Vienna, Austria, in 1999 and 2004, respectively. From 2001 till 2004, he was with the Institute of Communications and Radio Frequency Engineering, Vienna University of Technology. Since 2004, he has been with BMW Group Research and Technology, Munich. His research interests are in

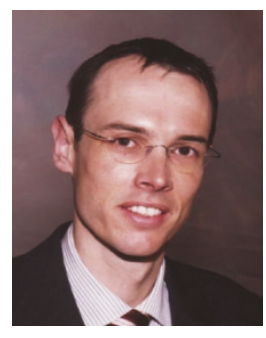
the areas of wireless communications, signal processing, and networking. 